\title{
Analyse diallèle et intégration de la sensibilité à la rouille courbeuse dans le programme d'amélioration du pin maritime
}

\author{
P Baradat ${ }^{*}$, ML Desprez-Loustau² \\ ${ }^{1}$ Inra et unité de recherche biométrie du Cirad-CP, 2477, avenue du Val-de-Montferrand, BP 5035 , \\ 34032 Montpellier cedex 1; \\ ${ }^{2}$ Station de pathologie végétale, centre de recherches Inra de Bordeaux, 71, avenue E-Bourleaux, \\ $B P$ 81, 33883 Villenave-d'Omon cedex, France
}

(Reçu le 27 avril 1995 ; accepté le 5 août 1996)

\begin{abstract}
Summary - Diallel analysis and integration in the breeding program of maritime pine of sensitivity to twisting rust. Maritime pines of the Landes provenance from two diallel mating designs have been studied between the third and fifth growing seasons for sensitivity to twisting rust (Malampsora pinitorqua), growth and phenology. The best criterion for sensitivity to twisting rust is the percentage of shoots with at least one canker occurring in the same growing season. Expression of rust sensitivity by this trait is partly dependent on vigour (positive correlation) and lateness (negative correlation). However, the major part of between-families variability for percentage of attacked shoots may be related to the original behaviour for this trait, uncorrelated to vigour and phenology. Genetic analysis of sensitivity has shown that the part of variability attributable to maternal effects is unsignificant. The same is true for genetic variability of dominance effects. On the other hand, additive genetic variability is great and highly significant. This variability would enable to realize important genetic gains for Melampsora resistance in Landes origin of maritime pine. Genetic correlation between rust sensitivity and polycyclism seems to be non-existent. Genetic correlation between rust sensitivity and juvenile growth expressed by 5 years height is not stable. It has a minor potential influence on integration of rust resistance criterion in the breeding program of maritime pine where volume growth is an important goal.
\end{abstract}

Melampsora pinitorqua / rust / maritime pine / diallel / selection / resistance

Résumé - Des pins maritimes de provenance landaise issus de deux plans de croisements diallèles ont été étudiés à des âges variant de la troisième à la cinquième saison de végétation pour la sensibilité à la rouille courbeuse (Melampsora pinitorqua), la croissance et la phénologie. Le meilleur critère de sensibilité à la rouille courbeuse est le pourcentage de pousses, formées lors de l'année de l'attaque, présentant au moins un chancre. L'expression de la sensibilité par ce critère est influencée par la vigueur (liaison positive) et la tardiveté (liaison négative). Toutefois, l'essentiel de la variabilité familiale pour le pourcentage de pousses attaquées est le reflet de caractéristiques propres, indépendantes de la vigueur et de la phénologie. L'analyse génétique de la sensibilité a montré que la part de la variabilité due à des effets maternels n'est pas significative. Il en est de même pour la variabilité génétique des effets de dominance. En revanche, la variabilité génétique d'origine additive se révèle notable et

* Correspondance et tirés à part Tél : (33) 0467615972 ; fax : (33) 0467617183 ; courriel : baradat@cirad.fr 
très significative. Cette variabilité autoriserait des gains génétiques importants pour la résistance à Melampsora du pin maritime des Landes. Il ne semble pas exister de liaison génétique entre sensibilité à la rouille et polycyclisme. La corrélation génétique entre sensibilité et vigueur juvénile, exprimée par la hauteur à cinq ans, est instable et ne peut avoir que peu d'incidence sur l'intégration du critère de sensibilité à la rouille dans le programme d'amélioration du pin maritime pour lequel la croissance en volume est un objectif important.

Melampsora pinitorqua / rouille / pin maritime / diallèle / sélection / résistance

\section{INTRODUCTION}

La rouille courbeuse des pins dont l'agent est Melampsora pinitorqua Rostr peut causer des dommages sévères aux jeunes semis de pin maritime, dans les 5 premières années, compromettant la valeur d'avenir du peuplement. Son impact économique est susceptible de s'accrô̂tre du fait de l' augmentation de la proportion de reboisements installés par plantation dans le massif landais (à peu près $50 \%$ actuellement). Dans ce cas, une densité initiale de 1200 plants/hectare ou moins rend difficile une correction sylvicole par élimination des plants les plus atteints à la suite d'une attaque massive. Ceci rend très importante la recherche d'une lutte contre la rouille, notamment par la création de variétés résistantes. Dans un premier article (Desprez-Loustau et Baradat, 1991), il a été montré que la variabilité de la sensibilité à la rouille courbeuse entre hybrides interraciaux est notable et s'explique essentiellement par des différences de précocité et de vigueur des plants au moment de la dissémination des basidiospores émises par l'hôte alternant (Populus tremula).

Il nous est apparu intéressant de rechercher s'il existe également une variabilité de la sensibilité à la rouille au sein de la race landaise ainsi qu'éventuellement si les causes de cette variabilité sont liées à la précocité et à la vigueur. Par ailleurs, il était important de vérifier si l'on pouvait ou non intégrer la sélection pour une meilleure résistance à la rouille courbeuse dans le programme d'amélioration du pin maritime. Ce programme est en effet essentiellement fondé sur une sélection récurrente à l'intérieur de la race landaise (Baradat et Pastuszka, 1992). L'héritabilité de la résistance à la rouille a été étudiée chez le pin sylvestre (Klingström, 1969 ; Weissenberg, 1978 ; Martinsson, 1975.
1980, 1987). Chez le pin maritime, on dispose de quelques résultats non publiés résultant d'observations sur des familles de pleins-frères installées en verger à graines.

Le présent travail aborde ces deux aspects, phytopathologique et génétique, à travers l'analyse de deux tests de descendances issus de plans de croisements diallèles. Ce type de plan de croisements se prête bien à l' analyse génétique et, notamment, à la détermination de la part de variabilité due aux effets maternels dont l'influence a été notée chez le pin sylvestre (Martinsson, 1980).

\section{MATÉRIEL ET MÉTHODES}

\section{Matériel}

\section{Matériel végétal}

Deux plans de croisements diallèles avec croisements réciproques et autofécondations ont été utilisés. Un diallèle $6 \times 6$ et un diallèle $12 \times 12$ ayant respectivement 6 et 29 combinaisons absentes. Les deux jeux de parents sont différents; il y a donc 18 parents au total. Il s'agit de clones $\mathrm{G}_{0}$ sélectionnés dans le massif landais sur des critères de croissance et de forme (Illy, 1966) et qui font partie de la population d'amélioration du pin maritime. Les croisements contrôlés ont été réalisés en parc à clones à la station de recherches forestières de Bordeaux-Cestas, au printemps 1978 pour le premier diallèle et au printemps 1980 pour le second. Pour ce dernier, les 12 parents représentent deux groupes de six clones, l'un monocyclique et l'autre polycyclique d'après leur valeur propre en test clonal et les caractéristiques de leurs descendants.

\section{Inoculum}

L'inoculum naturel de rouille a été renforcé, seulement sur le diallèle $6 \times 6$, en plantant ré- 
gulièrement sur chaque ligne des trembles du clone 117-50-1, très sensible à Melampsora pinitorqua (Desprez, 1980), à raison d'un tremble pour 18 pins maritimes. Les trembles ont été inoculés pendant l'été 1982 par pulvérisation d'une suspension aqueuse d'éciospores récoltées sur des pousses infectées de jeunes pins maritimes (20 000 éciospores $/ \mathrm{mL}$ ). Les plants ont été ensachés sous poche plastique pendant une nuit. En ce qui concerne le diallèle $12 \times 12$, l'inoculum naturel a été jugé suffisant du fait de la présence d'une forte densité de trembles sur son site d'implantation.

\section{Méthodes}

\section{Dispositifs expérimentaux}

Les plantations comparatives ont été réalisées à partir de plants élevés en pépinière pendant une saison de végétation, à la fin de l'hiver 1980 pour le matériel issu du diallèle 6 x 6 et à l'automne 1982 en ce qui concerne les plants appartenant au diallèle $12 \times 12$.

Les deux dispositifs sont installés dans la commune de Cestas (Gironde), en Lande mésophile, dans deux parcelles distinctes. Ils sont tous deux établis à la densité de 2272 arbres/hectare (espacement de $4 \mathrm{~m}$ entre les lignes et de $1,10 \mathrm{~m}$ sur la ligne).

Les parcelles unitaires sont linéaires, respectivement de 6 plants et de 4 plants. Les dispositifs statistiques sont les suivants :

- pour le diallèle $6 \times 6$, dispositif en blocs incomplets à composition aléatoire (Baradat, 1989) à 24 parcelles unitaires par bloc. Le nombre de répétitions par famille varie de 4 à 10 ;

- pour le diallèle $12 \times 12$, dispositif à 74 blocs incomplets à composition aléatoire à 16 parcelles unitaires par bloc. Le nombre de répétitions par famille varie de 4 à 15 . Ces 74 blocs ont été regroupés en 22 macroblocs sur des critères de voisinage.

\section{Observations et caractères dérivés}

Les observations ont été réalisées en juin 1983 et mai-juin et septembre 1984 pour le diallèle 6 x 6 et en septembre 1984 et octobre 1986 pour le diallèle $12 \times 12$.

\section{Caractères de croissance}

En désignant par $\mathrm{HT}_{i}$ la hauteur totale à un âge donné, $i$; par $\mathrm{PP}_{i}$, la longueur de pousse primaire ; et par $\mathrm{PS}_{i}$, celle de la pousse secondaire, les variables suivantes ont été créées :

- pour le diallèle $6 \times 6, \mathrm{HT}_{5}, \mathrm{PP}_{4}$, diamètre à la base de la pousse primaire de cinquième année. $\mathrm{DP}_{5}$ et élongations de la pousse primaire jusqu'au 9/05 (DP (5.1 $\left._{1}\right)$, du 9/05 au 17/05 (DP 5.2$)$, du $17 / 05$ au $28 / 05\left(D_{5.3}\right)$ et du 28/05 au 6/06 $\left(\mathrm{DP}_{5.4}\right)$;

- pour le diallèle $12 \times 12, \mathrm{HT}_{5}, \mathrm{PP}_{4}, \mathrm{PP}_{5}, \mathrm{PS}_{4}$, $\mathrm{PS}_{5}$ et degré de polycyclisme en quatrième et cinquième saisons de végétation :

$$
\mathrm{PC}_{+.5}=100\left(\mathrm{PS}_{4}+\mathrm{PS}_{5}\right) /\left(\mathrm{HT}_{5}-\mathrm{HT}_{3}\right) .
$$

\section{Caractères de phénologie de cinquième année (diallèle 6.6)}

Une note de phénologie de la pousse terminale a été attribuée à trois dates : 9/05 $\left(\mathrm{PH}_{5.1}\right), 17 / 05$ $\left(\mathrm{PH}_{5.2}\right)$, et $28 / 05\left(\mathrm{PH}_{5.3}\right)$. Les six valeurs possibles, de 0 à 5 , étaient croissantes avec la précocité et repérées par des stades précis de débourrement (Desprez-Loustau et Dupuis, 1994). Nous avons défini une note de phénologie moyenne pour ces trois dates définie par:

$$
\mathrm{PH}_{5}=\left(\mathrm{PH}_{5.1}+\mathrm{PH}_{52}+\mathrm{PH}_{5.3}\right) / 3 .
$$

Caractères destinés à évaluer le degré

d'infection.

En désignant par $\mathrm{NLI}_{i}$ le nombre de pousses latérales infectées en juin l'année $i$; par $\mathrm{NAF}_{i}$, la note d'attaque de la flèche $(0=$ indemne, $l=$ attaquée) ; par $\mathrm{NLT}_{i}$, le nombre de pousses latérales à la même date; par $\mathrm{NCF}_{i}$, le nombre de chancres sur la flèche, nous avons étudié :

- pour le diallèle $6 \times 6, N_{C F}$ et pourcentages de pousses infectées lors des attaques de quatrième et de cinquième années, la flèche étant comprise dans le nombre de pousses :

$$
\mathrm{SP}_{+}=100\left(\mathrm{NAF}_{+}+\mathrm{NLI}_{4}\right) /\left(\mathrm{NLT}_{+}+1\right)
$$

et

$$
\mathrm{SP}_{5}=100\left(\mathrm{sgn}\left(\mathrm{NCF}_{5}\right)+\mathrm{NLI}_{5}\right) /\left(\mathrm{NLT}_{5}+1\right)
$$

où la fonction sgn prend la valeur 0 si son argument vaut 0 et 1 s'il est $>0$; 
- pour le diallèle $12 \times 12$, caractère en troisième année :

$$
\mathrm{SP}_{3}=100\left(\operatorname{sgn}\left(\mathrm{NCF}_{3}\right)+\mathrm{NLI}_{3}\right) /\left(\mathrm{NLT}_{3}+1\right) .
$$

Réaction de la flèche de cinquième année à l'infection (diallèle $6 \times 6$ )

$\mathrm{RF}_{5}$, notation de 1 à 3 de la réaction de la pousse terminale. Dégâts croissants avec la note, de la cicatrisation (1) à une déformation plus ou moins forte (2) et à la mort (3).

Nous avons également analysé les trois variables qualitatives correspondant à chacun de ses trois niveaux $(0$, non-appartenance à la catégorie, 1, appartenance), codées respectivement par $\mathrm{RF}_{5.1}, \mathrm{RF}_{5.2}$ et $\mathrm{RF}_{5.3}$.

\section{Analyse des dispositifs expérimentaux}

Les analyses de variance en classification croisée et en modèle mixte (effet bloc fixé et effet famille aléatoire) ont été réalisées selon le modèle « Henderson III » (Searle, 1971), avec une variante proposée par la librairie Opep (Baradat, 1989 ; Baradat et Labbé, 1995) permettant d'estimer les composantes individuelles de la variance ou de la covariance, en l'absence d'interaction, sans utiliser les sommes de carrés ou de coproduits intracellule. Cette modification est bien adaptée au traitement de variables discrètes, par exemple de type 0-1. Elle est décrite en annexe.

Les analyses en modèle diallèle ont été réalisées selon une procédure adaptée aux plans non-orthogonaux avec croisements réciproques et, éventuellement, des autofécondations. Elle est donnée par Garretsen et Keuls (1977) pour le modèle aléatoire et Keuls et Garretsen (1978) pour le modèle à effets fixés. Le modèle demidiallèle, que nous avons également utilisé, a été construit par simplification de l'analyse générale. Nous nous sommes intéressés seulement au modèle aléatoire. En effet, notre but était d'estimer les composantes de la variance et de la covariance dues aux aptitudes générales et spécifiques et aux effets maternels.

Le modèle mixte, avec un effet bloc fixé, s'écrit pour le diallèle complet, $\mu$ désignant la moyenne générale ; $a_{i}$ et $a_{j}$, les aptitudes générales à la combinaison des parents $i$ et $j ; s_{i j}$, l'aptitude spécifique à la combinaison du cou- ple $(i, j) ; \beta_{k}$, l'effet du bloc $k$ et $e_{i j k l}$, la déviation individuelle à la moyenne de famille ajustée au bloc :

$$
y_{j k l}=\mu+a_{i}+a_{j}+s_{i j}+d_{i}+d_{j}+r_{i j}+\beta_{k}+e_{i j k l}
$$

et, pour le demi-diallèle :

$$
y_{i j k l}=\mu+a_{i}+a_{j}+s_{i j}+\beta_{k}+e^{\prime}{ }_{i k i}
$$

Les nouveaux résidus du modèle sont apurés de l'effet bloc mais intègrent d'éventuelles interactions bloc-famille.

Le tableau I présente l'analyse de variance du modèle mixte correspondant au diallèle complet sans autofécondations.

Les espérances des carrés moyens sont données dans le cas général et l'expression des coefficients des variances est trop complexe pour être détaillée dans cet article. Ce modèle est différent de celui donné par Griffing (1966) pour un plan diallèle complet avec croisements réciproques sans autofécondations, tel qu'il est repris sans modification par Becker (1984). Les espérances convergent dans le cas orthogonal équilibré, avec celles données par Cockerham (1963). Ce dernier auteur montre par ailleurs que le modèle qui sera repris par Griffing en 1966 redéfinit les composantes de la variance correspondant aux effets génétiques et aux effets réciproques, effets réciproques généraux et spécifiques confondus, de la façon suivante:

$$
\begin{gathered}
\sigma_{d i}^{2}=\sigma_{a}^{2}+(1 / 2) \sigma_{i l}^{2}, \sigma_{s^{2}}^{2}=(1 / 2)\left(\sigma_{r}^{2}+2 \sigma_{s}^{2}\right) \\
\text { et } \sigma_{i^{\prime}}^{2}=\sigma_{i}^{2}+2 \sigma_{d}^{2}
\end{gathered}
$$

Le modèle présenté par Gallais (1990) est directement dérivé de celui de Griffing mais isole les deux variances des effets réciproques, $\sigma^{2}$, et $\sigma^{2}{ }^{2}$. Les deux approches permettent des estimations non biaisées des variances d'AGC et d'ASC si les variances des effets réciproques sont nulles. Ce n'est que dans un schéma orthogonal, ou, au minimum, avec un plan de croisements équilibré, que les tests $F$ des effets aléatoires peuvent être utilisés en toute rigueur. Par la suite, nous n'utiliserons donc pas de tests $F$ pour établir la signification des variances des effets du modèle diallèle. En revanche, l'estimation des composantes de la variance et de la 
covariance est sans biais dans le cas général. L'estimation des composantes intrafamille sans utilisation des sommes de carrés et de coproduits individuels est possible mais n'a pas due être mise en œuvre compte tenu de la nature des caractères étudiés.

La variance totale, ou phénotypique, correspondant à l'analyse en modèle diallèle complet est donnée par :

$$
\sigma_{P}^{2}=\sigma^{2}\left(y_{i|k|}\right)=2 \sigma_{a}^{2}+\sigma_{t}^{2}+2 \sigma_{d}^{2}+\sigma_{r}^{2}+\sigma_{e}^{2}
$$

Pour l'analyse en modèle demi-diallèle, la variance intrafamille étant redéfinie de la façon indiquée ci-dessous, on a :

$$
\sigma_{p}^{2}=\sigma^{2}\left(y_{i j k i}\right)=2 \sigma_{i l}^{2}+\sigma_{s}^{2}+\sigma_{i}^{2}
$$

Le modèle génétique utilisé ne prend pas en compte les effets d'épistasie ; il implique en outre que le coefficient de consanguinité de la population dont sont issus les 18 parents soit nul. Cette dernière hypothèse est tout à fait réaliste dans le cas du pin maritime compte tenu des résultats obtenus sur les lois de croisements et la structuration génétique des peuplements aquitains (Baradat et Marpeau-Bezard, 1988 ; Baradat et al, 1995b ; Maillart, 1993). Dans ces conditions, la variance des effets génétiques additifs, $\sigma_{A}^{2}$, et la variance des effets de dominance, $\sigma_{b}^{2}$, sont données pour les deux types d'analyse par :

$$
\sigma_{i}^{2}=4 \operatorname{cov}(H S)=4 \sigma_{s}^{2}
$$

et

$$
\sigma_{t}^{2}=4[\operatorname{cov}(F S)-2 \operatorname{cov}(H S)]=4 \sigma_{,}^{2}
$$

avec $\operatorname{cov}(H S)=$ covariance entre demi-frères et $\operatorname{cov}(F S)=$ covariance entre pleins-frères.

La même règle vaut pour les covariances génétiques entre caractères différents. Dans les développements qui suivent, les héritabilités au sens strict et au sens large sont définie de façon

\begin{tabular}{|c|c|c|c|}
\hline $\begin{array}{c}\text { Carré moyen } \\
\text { et variance de l'effet }\end{array}$ & Degré de liberté & $\begin{array}{l}\text { Espérance du carré moyen : } \\
\text { E(CM) }\end{array}$ & Test $\mathrm{F}$ \\
\hline Bloc, $C M_{b}$ & $\mathrm{~B}-\mathrm{I}$ & $\sigma_{e}^{2}+\sum_{j=1}^{B} n_{. k .} \beta_{k}^{2} /(B-1)$ & $\begin{array}{l}C M_{b} / C M_{e} \\
\text { non biaisé }\end{array}$ \\
\hline $\begin{array}{l}\text { Aptitude générale, } A G C \\
C M_{a}, \sigma_{a}^{2}\end{array}$ & $P-1$ & $\sigma_{a}^{2}+k_{1} \sigma_{r}^{2}+k_{2} \sigma_{a}^{2}+k_{3} \sigma_{s}^{2}+k_{4} \sigma_{a}^{2}$ & $\begin{array}{l}C M a / C M_{s} \\
\text { biaisé }\end{array}$ \\
\hline $\begin{array}{l}\text { Aptitude spécifique, } A S C \\
C M_{s}, \sigma_{s}^{2}\end{array}$ & $\mathrm{C}-\mathrm{P}$ & $\sigma_{c^{\prime}}^{2}+k_{5} \sigma_{r}^{2}+k_{6} \sigma_{d}^{2}+k_{7} \sigma_{s}^{2}$ & $\begin{array}{l}C M_{\lrcorner} / C M_{r} \\
\text { biaisé }\end{array}$ \\
\hline $\begin{array}{l}\text { Effet réciproque général } \\
C M_{d}, \sigma_{d}^{2}\end{array}$ & $P-R-1$ & $\sigma_{r}^{2}+k_{8} \sigma_{r}^{2}+k_{9} \sigma_{d}^{2}+k_{10} \sigma_{s}^{2}$ & $\begin{array}{l}C M_{d} / C M_{r} \\
\text { biaisé }\end{array}$ \\
\hline $\begin{array}{l}\text { Effet réciproque spécifique } \\
C M_{r}, \sigma_{r}^{2}\end{array}$ & $D-C-P+R+1$ & $\sigma_{e}^{2}+k_{11} \sigma_{r}^{2}+k_{12} \sigma_{d}^{2}+k_{13} \sigma_{s}^{2}$ & $\begin{array}{c}C M_{n} / C M_{e} b \\
\text { biaisé }\end{array}$ \\
\hline Intrafamille $C M_{e}, \sigma_{e}^{2}$ & $\mathrm{~N}-\mathrm{D}-\mathrm{B}+1$ & $\sigma_{e}^{2}$ & \\
\hline \multicolumn{4}{|c|}{$\begin{array}{l}B: \text { nombre de blocs, } P: \text { nombre de parents, } C: \text { nombre de croisements, réciproques confondus } D: \text { nombre de } \\
\text { croisements, réciproques non confondus, } R: \text { nombre de parents utilisés dans un seul sens. } N: \text { nombre total de } \\
\text { plants. Le test } F \text { de la variance d'AGC est fait sur le } C M \text { d'ASC. Les tests } F \text { de la variance d'ASC et de la variance } \\
\text { de l'effet réciproque général sont faits sur le } C M \text { d'effet réciproque général. L'hypothèse de nullité de la variance } \\
\text { de l'effet réciproque spécifique est testée sur le } C M \text { intra-famille. L'examen des espérances des composantes de } \\
\text { la variance montre que les tests } F \text { de nullité des variances des effets du modèle diallèle sont biaisés dans le cas } \\
\text { général. }\end{array}$} \\
\hline
\end{tabular}
classique respectivement par :

$$
h_{s s}^{2}=\sigma_{A}^{2} / \sigma_{P}^{2} \text { et } h_{s f}^{2}=\left(\sigma_{A}^{2}+\sigma_{D}^{2}\right) / \sigma_{P}^{2}
$$

Tableau I. Schéma du modèle d'analyse diallèle complet et dispositif en blocs. 
Les coefficients de prédiction génétique, dont les propriétés sont définies ci-dessous, sont calculés sur le même principe que les héritabilités et les formules d'estimation complètes sont indiquées en regard des tableaux de résultats.

La variance intrafamille est estimée directement par le carré moyen intrafamille. Les quatre autres variances sont estimées, dans le cas général, en résolvant un système de quatre équations dont elles sont les inconnues. On a donc:

$$
\hat{\sigma}_{e}^{2}=C M_{c}
$$

et

$$
\left[\begin{array}{l}
\hat{\sigma}_{a}^{2} \\
\hat{\sigma}_{s}^{2} \\
\hat{\sigma}_{s t}^{2} \\
\hat{\sigma}_{r}^{2}
\end{array}\right]=\left[\begin{array}{llll}
k_{1} & k_{2} & k_{3} & k_{4} \\
k_{5} & k_{6} & k_{7} & 0 \\
k_{8} & k_{9} & k_{10} & 0 \\
k_{11} & k_{12} & k_{13} & 0
\end{array}\right]^{-1}\left[\begin{array}{c}
C M_{c}-C M_{c} \\
C M_{s}-C M_{r} \\
C M_{s}-C M_{r} \\
C M_{r}-C M_{r}
\end{array}\right]
$$

Les covariances correspondant aux cinq variances sont estimées de façon analogue, en remplaçant les carrés moyens par les coproduits moyens entre tous les couples de caractères.

Pour le modèle demi-diallèle, on supprime les deux lignes correspondant aux effets réciproques et les coefficients $k_{1}, k_{2}, k_{5}$ et $k_{6}$ du tableau $I$. L'analyse se fait alors en regroupant les couples de croisements réciproques et l'ensemble des sommes de carrés ou de coproduits des effets réciproques sont confondues avec la somme des carrés et coproduits intrafamille. Le nombre de degrés de liberté correspondant est naturellement augmenté de celui des deux carrés moyens des effets réciproques.

Les erreurs standard sur les coefficients de corrélation intraclasse, à l'exclusion des coefficients de prédiction génétique de l'analyse diallèle, ont été calculées par la formule approchée de Mode et Robinson (1959).

Nous avons utilisé la méthode du Jackknife (Lebart et al, 1979 ; Saporta, 1990), plus simple et plus robuste, pour évaluer les erreurs standard sur les estimations des composantes de la variance et des paramètres génétiques obtenues par l'analyse diallèle.

Le coefficient de prédiction génétique ou CPG (Baradat, 1976) a été conçu comme le développement de la notion d'héritabilité généralisée (Gallais, 1973)

Ce paramètre étend la notion d'héritabilité à deux caractères différents. Baradat (1989) montre comment la généralisation du $C P G$ à toute méthode de sélection peut se faire facilement en utilisant la théorie des index de sélection. Baradat et al (1995a) présentent une synthèse générale de la théorie des index de sélection en y intégrant la notion de sélection indirecte dans le cadre très général d'un modèle multicaractère. On peut définir un coefficient de prédiction génétique généralisé entre un caractère $x_{i}$ et un index de sélection quelconque, $I$, d'écart type $\sigma(l)$ et utilisant ce caractère (seul ou associé avec d'autres). On trouve que la formule donnant le $C P G$ généralisé est :

$$
\begin{aligned}
C P G\left({ }_{x . i}, I\right) & =\operatorname{cov}\left[G\left({ }_{x . i}\right), I\right] /\left[\sigma P\left({ }_{x . i}\right) \sigma(I)\right] \\
& =\sigma(I) / \sigma P\left({ }_{x . i}\right)
\end{aligned}
$$

Il ne reste plus alors qu'à exprimer $\sigma(I)$ dans chaque situation particulière.

Nous avons choisi, outre la sélection massale qui sert de référence, la sélection sur test de descendance, utilisée essentiellement pour la création variétale chez le pin maritime, et la sélection combinée sur familles de pleins-frères, qui a surtout vocation d'outil de gestion de la population d'amélioration.

Dans le cas de la sélection sur test de descendance utilisant des familles de demi-frères de $n$ individus issues de parents non consanguins, on obtient pour un index monocaractère, si le caractère sur lequel est fondé la sélection est $x_{i}$ :

\footnotetext{
"En fait, Masatoshi Nei (1960) avait déjà défini ce paramètre sous le nom de cohéritabilité (JA Vasquez, communication personnelle). Cette notion fut utilisée telle quelle par certains auteurs, par exemple Bedard et al (1971). Mais le coefficient fut redéfini par la suite comme le rapport de la covariance génétique à la covariance phénotypique (Rao et Goud, 1971 ; Janssens, 1979). Il a été utilisé sous cette nouvelle forme dans un grand nombre de références bibliographiques. Nei est donc le véritable père de la notion de régression standardisée du génotype sur le phénotype, mais le terme de cohéritabilité est ambigu à moins que l'on ne précise qu'il s'agit de la « cohéritabilité de Nei ».
} 


$$
\begin{gathered}
C P G\left[_{x, i}, I(x, i)\right] \\
=h^{2}\left(r, i_{i}\right) /\left[2 \sqrt{[(n-1) / 4] h^{2}\left(x_{i}\right)+1 \mid / n}\right]
\end{gathered}
$$

Si l'on veut obtenir le coefficient de prédiction génétique entre l'index construit avec le caractère $x_{i}$ et un autre caractère, $x_{j}$, on peut montrer qu'il suffit de remplacer, dans cette expression, $h^{2}\left(x_{i}\right)$ par $\left|C P G\left(x_{i}, x_{j}\right)\right|$ et de multiplier l'expression obtenue par un paramètre $\delta_{i j}$ tel que $\delta_{i j}=-1$ si $C P G<0$ et +1 si $C P G>0$. Dans le cas présent on peut éliminer $\delta_{i j}$, ce qui donne :

$$
\begin{gathered}
C P G\left[x j, I\left(x_{i}\right)\right]=C P G\left[x i, I\left(x_{j}\right)\right] \\
=C P G\left(x_{i}, x_{j}\right) /\left[2 \sqrt{|[(n-1) / 4]| C P G\left(x_{i}, x_{j}\right)|+1|} / \bar{n}\right]
\end{gathered}
$$

Cette formulation est symétrique, comme pour le coefficient de prédiction génétique simple qui ne s'applique qu'à la sélection massale. Elle est très utile pour comparer l'efficacité de diverses méthodes de sélection. En effet, la réponse corrélée d'un caractère ne dépend, pour une méthode de sélection et pour un effectif donné par unité génétique, que d'un seul paramètre. Une conséquence est que les propriétés du $C P G$ généralisé restent les mêmes que celles du $C P G$ simple. En particulier, l'efficacité de la sélection indirecte de $x_{j}$ par l'intermédiaire de $x_{i}$, par rapport à la sélection directe sur $x_{j}$, s'exprimera par :

$E R=\left|C P G\left[x_{j}, I\left(x_{i}\right)\right]-C P G\left[x_{j}, I\left(x_{j}\right)\right] / C P G\right| x_{j}, I\left(x_{j}\right) \mid$

Suivant le même principe que pour la sélection sur test de descendance, on obtient, pour $C P G\left[x_{j}, I\left(x_{i}\right)\right]$ concernant la sélection combinée sur familles de pleins-frères appartenant à un plan de croisements « single pair » (couples de parents déconnectés), l'équation 1.

Ces expressions peuvent être considérées comme des adaptations et des généralisations de celles données par Falconer (1981) pour différents types de sélection. Nous les utiliserons pour calculer les gains génétiques sur la résistance à Melampsora de l'origine landaise du pin maritime, par sélection directe sur $\mathrm{SP}_{3}$ ou $\mathrm{SP}_{5}$, et la réponse corrélée de la hauteur à cinq ans. Ce type de formalisation permet un traitement beaucoup plus simple de la sélection indirecte que par les autres méthodes proposées dans la littérature. En effet, on peut utiliser les formules de prédiction du gain génétique développées pour une sélection directe monocaractère.

\section{RÉSULTATS}

\section{Expression optimale de la sensibilité à Melampsora. Liaison avec phénologie et vigueur, définition du meilleur caractère prédicteur}

Nous avons procédé en quatre étapes :

- recherche de l'expression optimale des différences de sensibilité à Melampsora ;

- étude de la stabilité du caractère de sensibilité retenu à phénologie et vigueur constantes ;

- caractérisation de son pouvoir prédicteur vis à vis de la gravité de l'attaque ;

- analyse génétique proprement dite du caractère de sensibilité optimal.

Nous avons réalisé dans un premier temps une analyse de variance non-orthogonale à deux facteurs, bloc et famille, afin de déterminer quel modèle utiliser pour l'ajustement des données. Le tableau II donne le résultat de l'analyse de variance du diallèle $6 \times 6$ sur les deux caractères de sensibilité, les quatre caractères d'élongation en mai-juin 1984 , en cinquième année, $\Delta \mathrm{P}_{5.1}$ à $\Delta \mathrm{P}_{5.4}$ et la note de phénologie moyenne, $\mathrm{PH}_{5}$.

L'effet bloc est toujours hautement significatif, ce qui impose de travailler sur des données ajustées. L'effet famille est toujours très significatif, ce qui justifie l'analyse génétique. Enfin, la présence d'une interaction bloc $x$ famille, significative pour tous les caractères sauf $\Delta P_{5.4}$, rend préférable l'utilisation ultérieure d'un modèle d'analyse de covariance à deux facteurs, bloc et famille (Snedecor et Cochran, 1967).

\section{Équation 1.}

$$
C P G\left(x_{i}, x_{j}\right) \sqrt{1+\left[1-C P G\left(x_{i}, x_{j}\right)\right]^{2} / 4\left[\overline{1-\mid} C P G\left(x_{i}, x_{j}\right) \mid / 2^{\prime}(n-1) /\left[1+(n-1)\left|C P G\left(x_{i}, x_{j}\right)\right| / 2\right]_{\mid}^{i}\right.}
$$


Tableau II. Analyse de variance des caractères de vigueur et de phénologie du diallèle 6 x 6 .

\begin{tabular}{lcccccccc}
\hline $\begin{array}{l}\text { Source } \\
\text { de variation }\end{array}$ & $\begin{array}{c}\text { Degrés de } \\
\text { liberté }\end{array}$ & $S P_{4}$ & $S P_{5}$ & $\Delta P_{5,1}$ & $\Delta P_{5,2}$ & $\Delta P_{5,3}$ & $\Delta P_{5,4}$ & $P_{H_{5}}$ \\
\hline Bloc (fixé) & 9 & 570,77 & 11424 & 479,88 & 9,3750 & 25,349 & 11,389 & 1,2182 \\
& & 1,65 & 12,53 & 6,00 & 2,24 & 3,77 & 1,90 & 3,49 \\
& & 10,24 & 0,00 & 0,00 & 2,15 & 0,02 & 5,42 & 0,06 \\
Famille (aléatoire) & 29 & 561,01 & 3800,8 & 213,40 & 8,7946 & 21,298 & 10,752 & 1,1485 \\
& & 2,45 & 5,35 & 4,02 & 2,58 & 4,70 & 1,73 & 4,06 \\
& & 0,01 & 0,00 & 0,00 & 0,00 & 0,00 & 1,18 & 0,00 \\
Interaction & 186 & 354,14 & 911,39 & 79,916 & 4,1906 & 6,7261 & 5,9997 & 0,3493 \\
bloc x famille & & 1,51 & 1,28 & 1,51 & 1,23 & 1,48 & 0,96 & 1,24 \\
(aléatoire) & & 0,03 & 1,89 & 0,03 & 4,30 & 0,05 & 60,85 & 4,00 \\
& & & & & & & & \\
Intraparcelle & 452 & 228,97 & 709,89 & 53,017 & 3,4080 & 4,5289 & 6,2205 & 0,2829 \\
\hline
\end{tabular}

Ligne 1 : carré moyen ; ligne 2 : test $F$; ligne 3 : probabilité $\mathrm{F}>\mid \mathrm{H}_{\|}$(en \%).

C'est également ce modèle qu'il convient d'utiliser afin de trouver un critère objectif de choix entre $\mathrm{SP}_{4}$ et $\mathrm{SP}_{5}$ pour l'expression de la sensibilité à Melampsora.

Le tableau III résume les résultats permettant de comparer la valeur informative de $\mathrm{SP}_{4}$ et $\mathrm{SP}_{5}$. On constate tout d'abord que le pourcentage moyen de pousses infectées est environ trois fois plus élevé en cinquième année qu'en quatrième. Par ailleurs, le coefficient de corrélation intraclasse est également multiplié par un facteur voisin de trois. La différence est significative au seuil de $1 \%$.

Ceci peut être mis en relation avec une plage de variabilité et un écart type de l'effet famille pour la sensibilité beaucoup plus importants en cinquième année qu'en quatrième. Toutefois, la corrélation de rang de Spearman élevée $(0,79)$ entre les moyennes de familles pour les deux caractères montre qu'ils obéissent en grande partie à un déterminisme biologique identique. L'expression des différences entre familles est simplement meilleure en cinquième année.

Le caractère de base retenu pour la suite des analyses est donc $\mathrm{SP}_{5}$. Nous avons ensuite étudié dans quelle mesure les différences de sensibilité entre familles en cinquième année peuvent être expliquées par une variabilité affectant la tardiveté ou la vitesse d'élongation de la pousse primaire. Le tableau IV donne les résultats de l'analyse de covariance descendante multiple de $\mathrm{SP}_{5}$ (variable expliquée) avec les sept variables explicatives suivantes : longueur de la pousse primaire en quatrième année $\left(\mathrm{PP}_{4}\right)$ diamètre de la pousse primaire en cinquième année $\left(\mathrm{DP}_{5}\right)$ les quatre variables de croissance de cette pousse $\left(\Delta \mathrm{P}_{5.1}\right.$ à $\left.\Delta \mathrm{P}_{5.4}\right)$ et la note de phénologie moyenne $\left(\mathrm{PH}_{5}\right)$. Les corrélations

Tableau III. Comparaison du pourcentage de pousses infectées par Melampsora en quatrième et cinquième années.

\begin{tabular}{ccccc}
\hline & $\begin{array}{c}\text { \% de pousses } \\
\text { infectées }\end{array}$ & $\begin{array}{c}\text { Plage } \\
\text { de variation }\end{array}$ & $\begin{array}{c}\text { Ecart type } \\
\text { de l'effet famille }\end{array}$ & $\begin{array}{c}\text { Corrélation } \\
\text { intraclasse }\end{array}$ \\
\hline $\mathrm{SP}_{4}$ & 11,37 & $1,79-20,99$ & 3,13 & $0,035(0,016)$ \\
$\mathrm{SP}_{5}$ & 31,90 & $14,40-66,50$ & 11,43 & $0,144(0,033)$ \\
\hline
\end{tabular}

Les coefficients de corrélation intraclasse sont suivis de leur erreur standard entre parenthèses. Coefficient de corrélation de rang de Spearman : $0,790^{* *}$; corrélations intraclasse : $\left.t=\sigma_{f}^{2} / \mid \sigma_{f}^{2}+\sigma_{(h)}^{2}+\sigma_{e}^{2}\right] ;$ comparaison des corrélations intraclasse : $T=(0,144=0,035) / \sqrt{0,016^{2}+0,033^{2}}=2,97 * * ; * *$ significatif au seuil de $1 \%$. 
Tableau IV. Analyses de covariance simples et multiples du pourcentage de pousses infectées en cinquième année (variable expliquée) avec sept variables explicatives de croissance et de phénologie.

\begin{tabular}{|c|c|c|c|c|c|}
\hline & Palier & $\begin{array}{c}\text { Degrés } \\
\text { de liberté }\end{array}$ & $\begin{array}{l}\text { Carré } \\
\text { moyen }\end{array}$ & $\begin{array}{c}\text { Test } \\
\mathrm{F}\end{array}$ & $\begin{array}{c}P r o b \\
\mathrm{~F}>\mid H_{0}(\%)\end{array}$ \\
\hline \multicolumn{6}{|l|}{ Sept variables explicatives } \\
\hline Contribution totale & 1 & 7 & 10755 & 19,49 & 0,00 \\
\hline Contribution totale & 2 & 6 & 12456 & 22,73 & 0,00 \\
\hline Variable $\Delta \mathrm{P}_{5.3}(r=0,17)$ & & 1 & 7,8574 & 0,01 & 90,10 \\
\hline Contribution totale & 3 & 5 & 15050 & 27,27 & 0,00 \\
\hline Variable $\Delta \mathrm{P}_{5.2}(r=0,25)$ & & 1 & 25,251 & 0,05 & 82,54 \\
\hline Contribution totale & 4 & 4 & 18718 & 33,92 & 0,00 \\
\hline Variable $\mathrm{PP}_{4}(r=0,28)$ & & 1 & 375,11 & 0,68 & 58,48 \\
\hline Contribution totale & 5 & 3 & 24757 & 44,86 & 0,00 \\
\hline Variable $\mathrm{DP}_{5}(r=0,35)$ & & 1 & 601,81 & 1,09 & 29,74 \\
\hline Contribution totale & 6 & 2 & 36804 & 68,69 & 0,00 \\
\hline Variable $\Delta \mathbf{P}_{5,4}(r=0,09)$ & & 1 & 654,53 & 1,20 & 27,26 \\
\hline Variable $\Delta \mathbf{P}_{5.1}(r=0,43)$ & 7 & 1 & 60737 & 110,05 & 0,00 \\
\hline Variable $\mathrm{PH}_{5}(r=0,37)$ & & 1 & 12870 & 23,32 & 0,00 \\
\hline \multicolumn{6}{|l|}{ Test des effets ajustés } \\
\hline Famille & & 29 & 3197,7 & 5,54 & 0,00 \\
\hline Interaction bloc-famille & & 186 & 827,36 & 1,43 & 0,13 \\
\hline Erreur 1 (contri var) & & 445 & 551,89 & & \\
\hline Erreur 2 (effets ajustés) & & 451 & 576,80 & & \\
\hline \multicolumn{6}{|l|}{ Une variable: $\Delta P_{5 . I}$} \\
\hline Variable $\Delta \mathrm{P}_{5.1}$ & 1 & 1 & 60737 & 105,30 & 0,00 \\
\hline Test des effets ajustés & & & & & \\
\hline Famille & & 29 & 3197,7 & 5,54 & 0,00 \\
\hline Interaction bloc-famille & & 186 & 827,36 & 1,43 & 0,13 \\
\hline Erreur ( 1 et 2$)$ & & 451 & 576,80 & & \\
\hline \multicolumn{6}{|l|}{ Une variable : $\mathrm{PH}_{5}$} \\
\hline Variable $\mathrm{PH}_{5}$ & 1 & 1 & 44681 & 72,96 & 0,00 \\
\hline \multicolumn{6}{|l|}{ Test des effets ajustés } \\
\hline Famille & & 29 & 2406,3 & 3,93 & 0,00 \\
\hline Interaction bloc-famille & & 186 & 765,01 & 1,25 & 3,32 \\
\hline Erreur ( 1 et 2$)$ & & 451 & 612,40 & & \\
\hline \multicolumn{6}{|c|}{ Deux variables : $\triangle P_{5.1}$ et $P_{5}$} \\
\hline Variables $\Delta \mathrm{P}_{5.1}$ et $\mathrm{PH}_{5}$ & 1 & 2 & 36804 & 66,98 & 0,00 \\
\hline \multicolumn{6}{|l|}{ Test des effets ajustés } \\
\hline Famille & & 29 & 2559,9 & 4,66 & 0,00 \\
\hline Interaction bloc-famille & & 186 & 714,32 & 1,30 & 1,46 \\
\hline Erreur ( 1 et 2$)$ & & 450 & 549,48 & & \\
\hline
\end{tabular}

Entre parenthèses sont indiquées les corrélations au niveau intraparcelle unitaire entre $\mathrm{SP}_{5}$ (variable expliquée) et chaque variable explicative. 
Tableau V. Comparaison des coefficients de corrélation intraclasse et valeur des coefficients de rang des 30 familles du diallèle $6 \times 6$ pour le pourcentage de pousses attaquées en cinquième année, SP 5 non ajusté ou ajusté à la vigueur et/ou à la note de phénologie.

\begin{tabular}{|c|c|c|c|c|}
\hline & $\begin{array}{l}\text { Corrélation } \\
\text { interclasse } \mathrm{t}\end{array}$ & $\begin{array}{c}\text { ES de l'estimation } \\
\text { de } \mathrm{t}\end{array}$ & $\begin{array}{c}\text { Test diff de val de } \mathrm{t} \\
\text { avec } S P_{5}\end{array}$ & $\begin{array}{l}\text { Corrélation de } \\
\text { rang avec } S P_{5}\end{array}$ \\
\hline $\mathrm{SP}_{5}$ non ajusté & 0,144 & 0,033 & -------- & 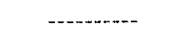 \\
\hline $\mathrm{SP}_{5}$ ajusté à $\mathrm{PH}_{5}$ & 0,120 & 0,027 & $0,56 \mathrm{NS}$ & $0,938 * *$ \\
\hline $\mathrm{SP}_{5}$ ajusté à $\Delta \mathrm{P}_{5}$ & 0,135 & 0,031 & $0,36 \mathrm{NS}$ & $0,970 * *$ \\
\hline $\mathrm{SP}_{5}$ ajusté à $\mathrm{PH}_{5}$ et $\Delta \mathrm{P}_{5}$ & 0,117 & 0,027 & $0,44 \mathrm{NS}$ & $0,929 * *$ \\
\hline
\end{tabular}

NS : non significatif au seuil de $40 \%$; ** significatif au seuil de $1 \%$; coefficient de corrélation intraclasse : $t=\sigma_{f}^{2} /\left[\sigma_{f}^{2}+\sigma_{(h f)}^{2}+\sigma_{e}^{2}\right] ;$ test $T:\left|t_{1}-t_{2}\right| / \sqrt{E S_{1}^{2}+E S_{2}^{2}}$.

intra- parcelle unitaire entre $\mathrm{SP}_{5}$ et les sept variables explicatives sont données dans la première colonne du tableau. Elles sont toutes positives. La sensibilité à Melampsora est donc, à ce niveau, liée positivement à la vigueur et négativement à la tardiveté (la note de phénologie moyenne diminue quand la tardiveté augmente).

On constate que les deux seules variables explicatives à effet significatif sont, par ordre d'importance décroissante, $\Delta \mathbf{P}_{5.1}$ et $\mathrm{PH}_{5}$.

En vue d'étudier les propriétés génétiques du pourcentage de rameaux infectés ajusté, nous avons complété l'analyse de covariance en testant l'efficacité de l'ajustement par chaque covariable à effet significatif et, conjointement, par les deux.
Les résultats concernant les deux analyses de covariance simples et l'analyse de covariance double sont reportés en bas du tableau IV.

Nous avons ensuite examiné si l'ajustement aux deux variables explicatives à effet significatif, $\Delta \mathbf{P}_{5.1}$ et $\mathbf{P H}_{5}$, modifiait les caractéristiques du pourcentage de pousses attaquées en cinquième année, $\mathrm{SP}_{5}$.

Le tableau $\mathrm{V}$ montre que les propriétés de $\mathrm{SP}_{5}$ restent pratiquement inchangées, que l'ajustement porte sur une seule variable explicative ou sur les deux : en effet, les corrélations intraclasse ne sont pas significativement différentes et les corrélations de rang, pour le classement des 30 familles, entre $\mathrm{SP}_{5}$ non ajusté et $\mathrm{SP}_{5}$ ajus-

Tableau VI. Analyse de variance des caractères de sensibilité des plants, d'attaque, de diamètre et de réaction de la pousse primaire pour les plants du diallèle $6 \times 6$ présentant au moins un chancre sur la flèche.

\begin{tabular}{lcccccccc}
\hline $\begin{array}{l}\text { Source } \\
\text { de variation }\end{array}$ & $\begin{array}{c}\text { Degrés } \\
\text { de liberté }\end{array}$ & $S P_{5}$ & $R F_{5.1}$ & $R F_{5.2}$ & $R F_{5.3}$ & $R F_{5.4}$ & $N F_{5}$ & $D P_{5}$ \\
\hline Bloc (fixé) & $\mathrm{B}-1=9$ & 4672,9 & 0,16820 & 0,16890 & 0,21312 & 0,59374 & 11,503 & 13,119 \\
& & 7,19 & 0,69 & 0,83 & 1,26 & 0,96 & 4,17 & 3,35 \\
& & 0,00 & 71,66 & 59,28 & 26,19 & 52,22 & 0,01 & 0,11 \\
& & & & & & & & \\
Famille & $\mathrm{F}-1=29$ & 1671,7 & 0,35966 & 0,11620 & 0,21519 & 1,0335 & 4,8606 & 8,0279 \\
(aléatoire) & & 2,57 & 1,48 & 0,57 & 1,28 & 1,67 & 1,76 & 2,14 \\
& & 0,02 & 7,30 & 96,00 & 17,89 & 2,85 & 1,74 & 0,23 \\
Interaction & $\mathrm{C}-\mathrm{B}-\mathrm{F}+1$ & 649,49 & 0,24315 & 0,20405 & 0,16848 & 0,61921 & 2,7572 & 3,9184 \\
bloc-famille & $=126$ & 1,16 & 1,05 & 1,11 & 1,17 & 1,09 & 0,69 & 1,04 \\
(aléatoire) & & 21,16 & 40,03 & 27,65 & 19,65 & 32,36 & 97,79 & 40,38 \\
Intraparcelle & $\mathrm{N}-\mathrm{C}=119$ & 561,72 & 0,23221 & 0,18319 & 0,14426 & 0,56975 & 3,9770 & 3,7482 \\
\hline
\end{tabular}

Pour les lignes correspondant aux effets bloc, famille et interaction bloc-famille sont donnés de haut en bas le carré moyen, la valeur du test $F$ et la probabilité $F>\mathrm{H}_{0}$, en $\left.\%\right) . B$ : nombre de blocs $(10) ; F:$ nombre de familles (30) ; $C:$ nombre de parcelles unitaires $(165) ; N$ : nombre total de plants (284). 
té sont très élevées, toujours supérieures à 0,84 **.

Nous avons donc considéré, pour la suite de ce travail, que la sensibilité à Melampsora, traduite par le pourcentage de pousses attaquées, était une caractéristique originale de chaque plant et non le simple reflet de sa précocité ou de la vitesse d'élongation de ses pousses au moment de la dissémination des basidiospores. Nous concentrerons donc l'analyse du déterminisme génétique de la sensibilité au parasite sur ce caractère. Mais il convient auparavant de savoir quel est, au niveau familial, son pouvoir prédicteur vis-à-vis de l'intensité d'attaque de la pousse primaire et de l'importance de sa réaction.

Le tableau VI présente le résultat de l'analyse de variance croisant les facteurs bloc et famille pour les sept caractères suivants : la sensibilité $\left(\mathrm{SP}_{5}\right)$, les trois variables qualitatives de réaction de la pousse terminale à Melampsora $\left(\mathrm{RF}_{5.1}\right)$ à $\left(\mathrm{RF}_{5.3}\right)$, le degré de réaction moyen de la pousse, $\left(R_{5}\right)$, le nombre de chancres sur la pousse terminale $\left(\mathrm{NCF}_{5}\right)$ et son diamètre $\left(\mathrm{DP}_{5}\right)$. Seuls ont été considérés les 284 plants présentant au moins un chancre sur la pousse terminale $\left(\mathrm{NCF}_{5}>0\right)$ puisque la notation de réaction à l'attaque n'a de sens que sur ce sous-ensemble. Comme pour les autres analyses, l'effet bloc a été considéré comme fixé et l'effet famille comme aléatoire.

Mais, puisque l'interaction bloc-famille n'est jamais significative, les effets principaux ont été testés par rapport au carré moyen d'interaction qui est alors un estimateur sans biais de la variance intraparcelle unitaire.

Les effets d'interaction ont une distribution continue, qu'il s'agisse de variables qualitatives ou quantitatives. Cela a été vérifié graphiquement. Toutefois, ces distributions sont en général légèrement dissymétriques (coefficient de dissymétrie allant de $-0,217$ pour $\mathrm{RF}_{5.1}$, non significatif, à $1,07 * *$ pour $\mathrm{RF}_{5.3}$ ). La robustesse du test $F$ est suffisante vis-à-vis de cette dissymétrie modérée. Par la suite nous avons utilisé la technique du Jackknife (cf Matériel et méthodes) pour calculer les intervalles de confiance des paramètres dérivés de l'analyse de variance diallèle en modèle aléatoire. Cette méthode ne postule pas une normalité ni même une symétrie de la distribution des effets.

On constate que l'effet famille est significatif à un seuil de probabilité $<5 \%$ pour quatre caractères : $\mathrm{SP}_{5}, \mathrm{RF}_{5}, \mathrm{NCF}_{5}$ et $\mathrm{DP}_{5}$. C'est sur ces quatre caractères que portent les résultats du tableau VII qui présente les valeurs des coefficients de corrélation intraclasse entre plants de

Tableau VII. Valeurs des coefficients de corrélation intraclasse pour les quatre variables exprimant la sensibilité, l’intensité de l'attaque, la réaction et le diamètre de la flèche.

\begin{tabular}{|c|c|c|c|c|}
\hline & $S P_{5}$ & $R F_{5}$ & $\mathrm{NCF}_{5}$ & $D P_{5}$ \\
\hline $\mathrm{SP}_{5}$ & $\begin{array}{l}0,148 * * \\
0,040\end{array}$ & & & \\
\hline $\mathrm{RF}_{5}$ & $\begin{array}{l}0,086^{*} \\
0,038\end{array}$ & $\begin{array}{l}0,069^{*} \\
0,031\end{array}$ & & \\
\hline $\mathrm{NCF}_{5}$ & $\begin{array}{l}0,116^{* *} \\
0,036\end{array}$ & $\begin{array}{l}0,023 \mathrm{NS} \\
0,030\end{array}$ & $\begin{array}{l}0,078^{* *} \\
0,029\end{array}$ & \\
\hline DPs & $\begin{array}{l}0,010 \mathrm{NS} \\
0,041\end{array}$ & $\begin{array}{c}-0,024 \mathrm{NS} \\
0,034\end{array}$ & $\begin{array}{l}0,044 \mathrm{NS} \\
0,032\end{array}$ & $\begin{array}{l}0,104^{* *} \\
0,034\end{array}$ \\
\hline
\end{tabular}

Coefficient de corrélation intraclasse : $l^{(u n)}=\operatorname{cov}_{f}^{(t h)} /\left[\sqrt{\sigma_{f}^{3(h)}+\sigma_{i}^{2(h)}} \sqrt{\sigma_{f}^{2(h)}+\sigma_{i}^{2(n)}}\right]$; les caractères $l$ et $l$ peuvent être identiques ou différents. S'ils sont identiques, on retrouve l'expression classique d'un coefficient de corrélation intraclasse défini comme un rapport de variances. NS : non significativement différent de 0 au seuil de $5 \%$; *significativement différent de 0 au seuil de $5 \% ;{ }^{*}$ significativement différent de 0 au seuil de $1 \%$; sur la deuxième ligne figure l'erreur standard de l'estimation. 
la même famille de pleins-frères pour le même caractère ou pour deux caractères différents. Les erreurs standard des coefficients de corrélation intraclasse, $t$, confirment bien, pour les estimations de $t$ sur un seul caractère, les conclusions fondées sur les valeurs des tests $F$.

En effet, l'hypothèse nulle $\sigma_{f}^{2}=0$ est équivalente, dans ce cas, à $t=0$. On constate que le diamètre de la pousse primaire, $\mathrm{DP}_{5}$, n'a aucune valeur prédictive vis-à-vis de $\mathrm{RF}_{5}$ : la corrélation intraclasse entre les deux caractères est pratiquement nulle. En revanche, la corrélation intraclasse entre individus de la même famille pour les couples de caractères $\mathrm{SP}_{5}-\mathrm{RF}_{5}$ et $\mathrm{SP}_{5}-\mathrm{NCF}_{5}$ est au moins égale à leur corrélation intraclasse pour $\mathrm{RF}_{5}$ ou NCF 5 . D' autre part, le signe de ces corrélations intraclasses entre caractères différents est toujours positif. Cela signifie que la variable $\mathrm{SP}_{5}$ est, au niveau familial, un prédicteur de l'intensité de l'attaque sur la pousse primaire $\left(\mathrm{NCF}_{5}\right)$ ou de la réaction de la pousse terminale. $\left(\mathrm{RF}_{5}\right)$ au moins aussi bon que chacun de ces caractères. En conséquence, on peut considérer $\mathrm{SP}_{5}$ comme un caractère synthétique reflétant le comportement global des familles devant l'attaque de rouille, ceci à trois niveaux : probabilité et intensité d'attaque ainsi que réaction du plant après infection. Dans la mesure où nous pourrons montrer que les différences entre familles pour ce caractère sont bien d'origine génétique, il sera donc possible de fonder sur lui une stratégie de sélection pour une meilleure résistance du pin maritime des Landes à Melampsora.

\section{Analyse génétique de la sensibilité à Melampsora. Liaison avec la hauteur}

Nous analyserons le déterminisme génétique de ce caractère dans les deux essais, ainsi que sa liaison avec la vigueur. Le but est de juger s'il est

Tableau VIII. Paramètres génétiques et phénotypiques pour $\mathrm{SP}_{5}$ et $\mathrm{HT}_{5}$ (diallèle 6 × 6 ).

\begin{tabular}{|c|c|c|c|c|}
\hline & \multicolumn{2}{|c|}{ Paramètres génétiques de $\mathrm{SP}_{5}$} & \multicolumn{2}{|c|}{ Paramètres de réponse de $H T_{5}$} \\
\hline & Paramètres & Erreur standard & Paramètre & Erreur standard \\
\hline Variance d'AGC $\left(\sigma_{a}^{2}\right)$ & 63,119 & $\begin{array}{l}17,87 \\
3,53^{* *}\end{array}$ & & \\
\hline Variance d'ASC $\left(\sigma_{s}^{2}\right)$ & 6,230 & $\begin{array}{l}22,41 \\
0,28 \mathrm{NS}\end{array}$ & & \\
\hline Variance d'ERG $\left(\sigma_{l^{\prime}}^{2}\right)$ & 12,534 & $\begin{array}{l}9,94 \\
1,28 \mathrm{NS}\end{array}$ & & \\
\hline Variance d'ERS $\left(\sigma_{r}^{2}\right)$ & 0 & - & & \\
\hline Moyenne générale, $\mu$ & 33,32 & - & 164,51 & - \\
\hline $\mathrm{CV}$ phénotypique, $\sigma p / \mu^{\mathrm{a}}$ & 0,897 & - & 0,288 & - \\
\hline$C P G$ au sens strict $\left(C P G_{s s}\right)$ & 0,268 & $\begin{array}{l}0,068 \\
3,94 * *\end{array}$ & $-0,137$ & $\begin{array}{l}0,067 \\
2,14^{*}\end{array}$ \\
\hline
\end{tabular}

Pour $\mathrm{SP}_{5}$, le coefficient de prédiction génétique est l'héritabilité (prédiction de la valeur génétique du caractère

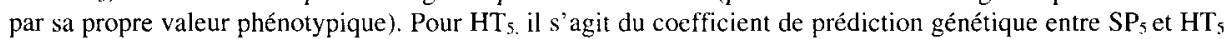
(prédiction de la valeur génétique de l'un des deux caractères par la valeur phénotypique de l'autre caractère). On a pour le même caractère (héritabilité) $: h_{w}^{2}=4 \sigma_{a l}^{2} /\left(2 \sigma_{u}^{2}+\sigma_{s}^{2}+2 \sigma_{\vec{l}}^{2}+\sigma_{r}^{2}+\sigma_{c}^{2}\right)$; pour deux caractères différents, $l$ et $l^{\prime}$ (coefficients de prédiction génétique) :

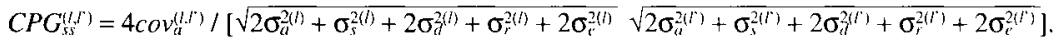

"Le coefficient de variation phénotypique, $\mathrm{CV}$, a été calculé par le rapport de l'écart type individuel des 663 plants issus d'allofécondation à leur moyenne, après ajustement à l'effet bloc ( 653 degrés de liberté). Comme la moyenne générale, $\mathrm{m}$, il est considéré comme connu et non estimé ; *significativement différent de 0 au seuil de $5 \%$; ${ }^{* *}$ significativement différent de 0 au seuil de $1 \%$; NS : non significatif au seuil de $20 \%$. 
utile d'inclure le critère de sensibilité au parasite dans le programme d'amélioration génétique du pin maritime des Landes et si la sélection doit porter sur la seule valeur génétique additive ou sur la valeur génétique totale de ce caractère. Par ailleurs, il importe de déterminer dans quelle mesure une telle sélection se ferait au détriment de la vigueur.

Le tableau VIII donne les estimations des variances d'aptitude générale et d'aptitude spécifique, ainsi que des effets réciproques généraux et spécifiques pour le diallèle $6 \times 6$. Vis-à-vis de chaque estimation est indiquée son erreur standard, calculée par la méthode du Jackknife avec $50^{\circ}$ de liberté. Les moyennes générales et les coefficients de variation phénotypiques des deux caractères, estimés sans utiliser le modèle diallèle, figurent également sur ce tableau en tant qu'éléments de calcul des gains génétiques.

On voit que, pour $\mathrm{SP}_{5}$, la seule variance élémentaire du modèle diallèle significativement différente de 0 est la variance d'AGC. Seul le calcul de l'héritabilité au sens strict a donc un sens. Elle est donnée, avec son erreur standard, sur la dernière ligne du tableau. Seul le coefficient de prédiction génétique au sens strict entre $\mathrm{SP}_{5}$ et $\mathrm{HT}_{5}$ a également un sens. Il est donné, avec son erreur standard et le test $T$ de signification, vis-à-vis de l'héritabilité au sens strict de $\mathrm{SP}_{5}$. Ces deux paramètres génétiques sont significativement différents de 0 . Le signe négatif du coefficient de prédiction génétique $(-0,137)$ indique un effet favorable sur la hauteur juvénile d'une sélection pour une moindre sensibilité à Melampsora.

Pour le diallèle $12 \times 12$ (tableau IX), les variances des effets réciproques ne sont pas significativement différentes de 0 et celle des effets d'aptitude spécifique à la combinaison est nulle. Seuls l'héritabilité au sens strict de $\mathrm{SP}_{3}$ et son coefficient de prédiction génétique avec $\mathrm{HT}_{5}$ ont donc un sens. Ces deux paramètres génétiques sont significativement différents de 0 .

On remarque toutefois que l'héritabilité de $\mathrm{SP}_{3}(0,057)$ est très inférieure à celle de $\mathrm{SP}_{5}$ $(0,268)$ et que son coefficient de prédiction génétique avec $\mathrm{HT}_{5}$ est, cette fois, positif $(0,035)$. Les tests $T$ de comparaison des deux héritabilités et des deux coefficients de prédic-

Tableau IX. Paramètres génétiques et phénotypiques pour $\mathrm{SP}_{3}$ et $\mathrm{HT}_{5}$ (diallèle $12 \times 12$ ).

\begin{tabular}{|c|c|c|c|c|}
\hline & \multicolumn{2}{|c|}{ Paramètres génétiques de $\mathrm{SP}_{3}$} & \multicolumn{2}{|c|}{ Paramètres de réponse de $H T_{5}$} \\
\hline & Paramètres & Erreur standard & Paramètre & Erreur standard \\
\hline Variance d' $A G C\left(\sigma_{a}^{2}\right)$ & 14,302 & $\begin{array}{l}3,74 \\
3,82^{* *}\end{array}$ & - & - \\
\hline Variance d' $A S C\left(\sigma_{s}^{2}\right)$ & 0 & - & - & - \\
\hline Variance d'ERG $\left(\sigma_{l l}^{2}\right)$ & 2,7955 & $\begin{array}{l}2,739 \\
1,02 \mathrm{NS}\end{array}$ & - & - \\
\hline Variance d'ERS $\left(\sigma_{r}^{2}\right)$ & 170,03 & $\begin{array}{c}359,73 \\
0,47 \mathrm{NS}\end{array}$ & - & - \\
\hline Moyenne générale, $\mu$ & 34,63 & - & 136,42 & - \\
\hline $\mathrm{CV}$ phénotypique, $\sigma p / \mu^{\mathrm{a}}$ & 0,835 & - & 0,274 & - \\
\hline$C P G$ au sens strict $\left(C P G_{s s}\right)$ & 0,057 & $\begin{array}{l}0,0092 \\
6,19 * *\end{array}$ & 0,035 & $\begin{array}{l}0,0104 \\
3,36^{* *}\end{array}$ \\
\hline
\end{tabular}

Pour $\mathrm{SP}_{3}$, le coefficient de prédiction génétique est l'héritabilité (prédiction de la valeur génétique du caractère par sa propre valeur phénotypique). Pour $\mathrm{HT}_{5}$, il s'agit du coefficient de prédiction génétique entre $\mathrm{SP}_{3}$ et $\mathrm{HT}_{5}$ (prédiction de la valeur génétique de l'un des deux caractères par la valeur phénotypique de l'autre caractère). L'expression de ces paramètres génétiques est donnée au bas du tableau VIII. "Le coefficient de variation phénotypique a été calculé par le rapport de l'écart type individuel des 2438 plants issus d'allofécondation à leur moyenne, après ajustement à l'effet bloc (2 416 degrés de liberté) ; comme la moyenne, $\mu$, il est considéré comme connu et non estimé ; *significativement différent de 0 au seuil de $5 \%$; **significativement différent de 0 au seuil de $1 \% ;$ NS : non significatif au seuil de $20 \%$. 
tion génétique valent respectivement 3,07 et 2,65 . Ils sont significatifs au seuil de $1 \%$.

On doit donc admettre que les deux caractères de sensibilité à Melampsora à 5 ans et à 3 ans, $\mathrm{SP}_{5}$ et $\mathrm{SP}_{3}$, avec des valeurs moyennes très voisines (respectivement 33,32 et $34,63 \%$ ), correspondent à des déterminismes génétiques qui ne sont pas identiques. Par ailleurs, la liaison génétique du caractère avec la hauteur à 5 ans change de signe entre les deux dispositifs. Le calcul de l'héritabilité au sens strict de $\mathrm{SP}_{4}$ dans le diallèle $6 \times 6$ et du coefficient de prédiction génétique entre $\mathrm{SP}_{4}$ et $\mathrm{HT}_{5}$ dans ce même essai est en faveur d'une évolution due à l'âge et pas seulement aux différences de croissance en hauteur entre les deux dispositifs. On obtient respectivement $0,068(\mathrm{ES}=0,046 ; T=1,48 \mathrm{NS})$ et $-0,072(\mathrm{ES}=0,053 ; T=1,36 \mathrm{NS})$. Toutefois, le faible pourcentage de pousses attaquées en quatrième année $(11,37 \%)$, présenté dans le tableau III, peut être en partie responsable de la faible héritabilité du caractère. Le problème de l'évolution, en fonction de l'âge au moment de l'attaque, de la corrélation génétique entre sensibilité à Melampsora et hauteur à 5 ans sera approfondi dans la discussion.

Le tableau $\mathrm{X}$ donne les coefficients de prédiction génétique entre $\mathrm{SP}_{3}$ et les accroissements primaires et secondaires en quatrième et en cinquième années ainsi qu'avec le degré de polycyclisme sur les deux années. On constate une indépendance entre $\mathrm{SP}_{3}$ et le degré de polycyclisme, $\mathrm{CP}_{4-5}$. En revanche, le coefficient de prédiction génétique entre $\mathrm{SP}_{3}$ et les accroissements primaires reste stable de la quatrième à la cinquième année : $T=0,83 \mathrm{NS}$. Le coefficient de prédiction génétique de $\mathrm{SP}_{3}$ avec les accroissements secondaires augmente de façon significa- tive entre la quatrième et la cinquième année : $T=3,70 * *$. On a donc un très léger antagonisme entre résistance à la rouille en troisième année et accroissement en hauteur en quatrième et cinquième saisons de végétation. L'examen de l'évolution comparée de l'accroissement primaire ou secondaire et du coefficient de prédiction génétique avec $\mathrm{SP}_{3}$ semble montrer que la valeur de ce paramètre est conditionnée par l'importance de l'accroissement moyen, indépendamment de sa nature, primaire ou secondaire. Ainsi, la seule valeur de $C P G$ non significativement différente de 0 correspond à un accroissement secondaire moyen très faible $(4,75 \mathrm{~cm})$.

Compte tenu de l'absence de variance d'effets réciproques dans la variance phénotypique de $\mathrm{SP}_{5}$ (diallèle $6 \times 6$ ) et $\mathrm{SP}_{3}$ (diallèle $12 \times 12$ ), il semblait judicieux de reprendre l'analyse de variance en modèle demi-diallèle en confondant les familles correspondant à des combinaisons réciproques. En effet, ce type d'analyse permet une estimation plus fiable des composantes d'AGC et d'ASC, en utilisant un modèle comportant le nombre minimum de paramètres. Le tableau XI donne les résultats de cette nouvelle analyse de variance. Est donnée également dans ce tableau l'héritabilité de $\mathrm{HT}_{5}$ qui intervient pour le calcul des gains génétiques corrélés (cf Matériel et méthodes). Les variances d'ASC ne sont toujours pas significativement différentes de 0. Les héritabilités des deux caractères de sensibilité à Melampsora sont légèrement augmentées ainsi que, en valeur absolue, celle des $C P G$ avec $\mathrm{HT}_{5}$.

On remarque que les coefficients de variation phénotypiques de SP3, SP5 et $\mathrm{HT}_{5}$ calculés à partir des composantes de la variance du modèle demi-diallèle sont très voisins de ceux estimés directement, sans analyse de variance, et donnés

Tableau X. Coefficients de prédiction génétique avec $\mathrm{SP}_{3}$ et coefficients de variation phénotypiques pour les cinq caractères de croissance primaire et secondaire (diallèle $12 \times 12$ ).

\begin{tabular}{|c|c|c|c|c|c|c|c|c|c|c|}
\hline & \multicolumn{2}{|c|}{$\mathrm{PP}_{4}$} & \multicolumn{2}{|c|}{$P P_{5}$} & \multicolumn{2}{|c|}{$\mathrm{PS}_{4}$} & \multicolumn{2}{|c|}{$P S_{5}$} & \multicolumn{2}{|c|}{$P C_{4-5}$} \\
\hline & Param & $E S$ & Param & $E S$ & Param & $E S$ & Param & $E S$ & Param & $E S$ \\
\hline$\mu$ & 18,36 & - & 41,44 & - & 4,75 & - & 16,42 & - & 75,70 & - \\
\hline$C P G_{s s}$ & 0,031 & $\begin{array}{c}0,010 \\
3,10^{* *}\end{array}$ & 0,045 & $\begin{array}{c}0,014 \\
3,21 * *\end{array}$ & $-0,007$ & $\begin{array}{c}0,010 \\
0,70 \mathrm{NS}\end{array}$ & 0.030 & $\begin{array}{c}0,010 \\
3,00 * *\end{array}$ & $-0,004$ & $\begin{array}{c}0,012 \\
0,33 \mathrm{NS}\end{array}$ \\
\hline
\end{tabular}

**Significativement différent de 0 au seuil de $1 \%$; NS : non significatif au seuil de $40 \%$. 
dans les tableaux VIII et IX. Ces derniers peuvent être considérés comme connus exactement. Le tableau XI donne les éléments de base pour le calcul des gains génétiques sur la résistance à la rouille courbeuse et les gains corrélés sur la hauteur à cing ans. Compte tenu de la remarque précédente, nous considérerons que les coefficients de variation phénotypiques qui y figurent sont connus sans erreur.

\section{Détermination des espérances de gains génétiques sur la sensibilité à Melampsora. Conséquence sur la croissance en hauteur juvénile.}

Nous avons donc montré que les variances des effets réciproques et d'aptitude spécifique peuvent être considérées comme nulles pour la sensibilité à Melampsora. Cette sensibilité est par ailleurs exprimée de façon optimale sur le diallèle $6 \times 6$, par le pourcentage de pousses attaquées pendant la cinquième saison de végétation. $\mathrm{La}$ transmission génétique de la sensibilité s'explique entièrement par un modèle additif, avec, pour un pourcentage de pousses attaquées de l'ordre de $30 \%$, une héritabilité forte à cinq ans dans cet essai et une héritabilité nettement plus faible à trois ans dans le diallèle $12 \times 12$. En chiffres ronds, on obtient des intervalles de confiance au seuil de $5 \%$ qui valent: $0,04<h 2<0,11$ (moyenne $=0,075)$ pour le diallèle $12 \times 12$ à trois ans et $0,17<h 2<0,42$ (moyenne $=0,295$ ) pour le diallèle $6 \times 6$ à cinq ans. Les intervalles de confiance correspondants des coefficients de prédiction génétique du caractère avec la hauteur à cinq ans ont les valeurs suivantes : $0,04<C P G<0,10$ (moyenne $=0,070$ ) et $-0,28<C P G<-0,09$ (moyenne $=-0,185)$. En partant des résultats

Tableau XI. Paramètres génétiques de $\mathrm{SP}_{5}$ (diallèle $6 \times 6$ ), $\mathrm{SP}_{3}$ (diallèle $12 \times 12$ ) et $\mathrm{HT}_{5}$, estimés par une analyse en demi-diallèle.

\begin{tabular}{|c|c|c|c|c|c|c|c|c|}
\hline & \multicolumn{4}{|c|}{ Diallèle $6 \times 6$} & \multicolumn{4}{|c|}{ Diallèle $12 \times 12$} \\
\hline & \multicolumn{2}{|c|}{$S P_{5}$} & \multicolumn{2}{|c|}{$H T_{5}$} & \multicolumn{2}{|c|}{$S P_{5}$} & \multicolumn{2}{|c|}{$H T_{5}$} \\
\hline & Param & $E S$ & Param & $E S$ & Param & $E S$ & Param & $E S$ \\
\hline CV phén, $\sigma_{\mathrm{p}} / \mu^{\mathrm{a}}$ & 0,926 & - & 0,295 & - & 0,841 & - & 0,276 & - \\
\hline $\operatorname{Var} \mathrm{d}^{\prime} A G C, \sigma_{a}^{2}$ & 69,26 & $\begin{array}{l}17,46 \\
3,97 * *\end{array}$ & - & - & 15,64 & $\begin{array}{l}3,62 \\
4,32 * *\end{array}$ & - & - \\
\hline $\operatorname{Var} \mathrm{d}^{\prime} A S C, \sigma_{S}^{2}$ & 5,10 & $\begin{array}{l}13,14 \\
0,39 \mathrm{NS}\end{array}$ & - & - & 4,69 & $\begin{array}{l}7,54 \\
0,62 \mathrm{NS}\end{array}$ & - & - \\
\hline$C P G_{s s}$ & 0,293 & $\begin{array}{l}0,062 \\
4,73 * *\end{array}$ & $-0,186$ & $\begin{array}{l}0,048 \\
3,87 * *\end{array}$ & 0,074 & $\begin{array}{l}0,016 \\
4,62 * *\end{array}$ & 0,071 & $\begin{array}{l}0,014 \\
5,07^{* *}\end{array}$ \\
\hline Héritabilité de $\mathrm{HT}_{5}$ & - & - & 0,414 & $\begin{array}{l}0,071 \\
5,83^{* *}\end{array}$ & - & - & 0,060 & $\begin{array}{l}0,014 \\
4,29 * *\end{array}$ \\
\hline
\end{tabular}

Pour $\mathrm{SP}_{3}$ et $\mathrm{SP}_{5}$, le coefficient de prédiction génétique est l'héritabilité (prédiction de la valeur génétique du caractère par sa propre valeur phénotypique). Pour $\mathrm{HT}_{5}$, il s'agit du coefficient de prédiction génétique entre $\mathrm{SP}_{3}$ et $\mathrm{HT}_{5}$ (prédiction de la valeur génétique de l'un des deux caractères par la valeur phénotypique de l'autre caractère). L'héritabilité de $\mathrm{HT}_{5}$ (dernière ligne du tableau) est également au sens strict. 'Le coefficient de variation phénotypique a été calculé par le rapport à la moyenne, $\mu$, de l'écart type phénotypique estimé en sommant les composantes de l'analyse en demi-diallèle. C'est la même valeur que celle qui figure au dénominateur de l'héritabilité. Pour le même caractère (héritabilité) : $h_{s s}^{2}=4 \sigma_{u}^{2} /\left(2 \sigma_{a}^{2}+\sigma_{s}^{2}+2 \sigma_{\epsilon}^{2}\right)$; pour deux caractères différents, $l$ et $l$ ' (coefficient de prédiction génétique) :

$C P G_{s s}^{(l, l)}=4 \operatorname{cov}_{a}^{(l, l)} /\left[\sqrt{2 \sigma_{a}^{2(h)}+\sigma_{s}^{2(l)}+2 \sigma_{c}^{2(l)}} \sqrt{2 \sigma_{a}^{2(r)}+\sigma_{s}^{2(r)}+2 \sigma_{e}^{2(l)}}\right] ; * *$ significativement différent de 0 au seuil de $1 \%$; NS : non significatif au seuil de $50 \%$. 
Tableau XII. Espérances de gains génétiques relatifs (en \%) pour la réduction de la sensibilité à Melampsora (contre-sélection pour ce caractère) et pour la hauteur à cinq ans, prédits à partir des paramètres génétiques estimés.

\begin{tabular}{|c|c|c|c|c|c|c|c|c|}
\hline & \multicolumn{2}{|c|}{ Gain asymptot } & \multicolumn{2}{|c|}{ Sélection massale } & \multicolumn{2}{|c|}{ Sélection sur TD } & \multicolumn{2}{|c|}{ Sélection comb } \\
\hline & $\mathrm{t}=50 \%$ & $\mathrm{t}=5 \%$ & $\mathrm{t}=50 \%$ & $\mathrm{t}=5 \%$ & $\mathrm{t}=50 \%$ & $\mathrm{t}=5 \%$ & $\mathrm{t}=50 \%$ & $\mathrm{t}=5 \%$ \\
\hline $\mathrm{SP}_{3 m i n}$ & 14,36 & 37,13 & 2,87 & 7,43 & 8,32 & 21,51 & 7,50 & 19,39 \\
\hline $\mathrm{SP}_{3 m o r}$ & 19,67 & 50,85 & 5,38 & 13,92 & 13,75 & 35,54 & 11,85 & 30,63 \\
\hline $\mathrm{SP}_{3 \text { max }}$ & 23,82 & 61,58 & 7,90 & 20,42 & 18,23 & 47,13 & 15,36 & 39,70 \\
\hline $\mathrm{SP}_{5 m i n}$ & 29,61 & 76,55 & 12,21 & 31,56 & 24,58 & 63,56 & 20,38 & 52,68 \\
\hline $\mathrm{SP}_{5 m \% y}$ & 39,01 & 100 & 21,18 & 54,77 & 35,04 & 90,16 & 28,96 & 74.86 \\
\hline $\mathrm{SP}_{5 \text { max }}$ & 46,54 & 100 & 30,16 & 77,98 & 41,02 & 100 & 36,47 & 94,29 \\
\hline $\mathrm{H}_{5} \mathrm{THT}_{5}$ & 15,14 & 39,14 & 9,58 & 24,76 & 13,94 & 36,03 & 11,77 & 30,42 \\
\hline $\mathrm{HT}_{5} \mid \mathrm{SP}_{3 m i n}$ & $-4,79$ & $-12,38$ & $-0,96$ & $-2,47$ & $-2,77$ & $-7,17$ & $-2,50$ & $-6,46$ \\
\hline $\mathrm{HT}_{5} \mathrm{ISP}_{3}$ moy & $-6,33$ & $-16,37$ & $-1,67$ & $-4,33$ & $-4,35$ & $-11,24$ & $-3,76$ & $-9,73$ \\
\hline $\mathrm{HT}_{5} \mid \mathrm{SP}_{3} \max _{x}$ & $-7,57$ & -19.57 & $-2,39$ & $-6,19$ & $-5,67$ & $-14,67$ & $-4,80$ & $-12,42$ \\
\hline $\mathrm{HT}_{5} \mathrm{ISP}_{5 \mathrm{~min}}$ & 7,18 & 18,57 & 2,15 & 5,57 & 5,25 & 13,58 & 4,48 & 11,60 \\
\hline $\mathrm{HT}_{5} \mathrm{ISP}_{5 m o 4}$ & 10,30 & 26,62 & 4,43 & 11,45 & 8,66 & 22,40 & 7,17 & 18,53 \\
\hline $\mathrm{HT}_{5} \mathrm{ISP}_{5 \text { max }}$ & 12,67 & 32,75 & 6,70 & 17,33 & 11,26 & 29,11 & 9,33 & 24,13 \\
\hline
\end{tabular}

$t:$ taux de sélection ; $m i n$ : borne inférieure du $C P G$ au seuil de $5 \%:$ moy : valeur moyenne arrondie du $C P G$; max : borne supéricure du $C P G$; les trois estimations de gain génétique pour la réduction de sensibilité à Melampsora, qui étaient légèrement supérieures à 100 , ont été tronquées puisque la vraie valeur ne peut évidemment pas dépasser 100 .

synthétiques du tableau XI et en utilisant les formules de prédiction du gain génétique développées dans la section Matériel et méthodes, nous pouvons évaluer les gains attendus par sélection massale, sur test de descendance, et combinée sur familles de pleins-frères. Ces trois méthodes de sélection sont facilement praticables dans le programme de sélection récurrente du pin maritime en Aquitaine, tel qu'il est conçu actuellement. Pour l'héritabilité de la hauteur à cing ans, nous prendrons la valeur moyenne arrondie de 0,4 qui est celle habituellement calculée en test de descendance (Bergoin, 1993). Enfin, pour le coefficient de variation phénotypique, nous adopterons 0,9 pour $\mathrm{SP}_{3}$ et $\mathrm{SP}_{5}$, moyenne arrondie entre les valeurs du tableau XI. Nous prendrons 0,3 pour le coefficient de variation de $\mathrm{HT}_{5}$, valeur arrondie légèrement supérieure à la moyenne des deux essais. Nous considérerons deux taux de sélection correspondant à deux objectifs différents : sélection faible, taux de $50 \%$ (gestion de la population d'amélioration), $i=0,798$ et sélection forte, taux de $5 \%$ (création d'une variété résistante), $i=2,063$.

Il est immédiat que l'espérance du gain génétique relatif maximum réalisable (asymptotique) par sélection directe sur le caractère $x_{i}$ est donnée par:

$$
\Delta G R_{\operatorname{mix}}\left(x_{i} \mid x_{i}\right)=i \sigma A\left(x_{i}\right) / \mu\left(x_{i}\right)=i C V\left(x_{i}\right) h\left(x_{i}\right)
$$

S'il s'agit d'une sélection indirecte du caractère $x_{j}$ par l'intermédiaire du caractère $x_{i}$, on obtient en définissant le paramètre $\delta_{i j}$ de la même façon que dans la section matériel et méthodes :

$$
\Delta G R_{\mathrm{max}}\left(x_{j} \mid x_{i}\right)=i C V\left(x_{j}\right) \delta_{i j} \sqrt{\left|C P\left(x_{i}, x_{j}\right)\right|}
$$

Ces gains génétiques sont ceux que l'on réaliserait avec une connaissance parfaite des valeurs génétiques additives du caractère sur lequel porte la sélection. On s'en rapproche avec une sélection sur test de descendance et des effectifs par famille très grands. Le tableau XII donne les gains génétiques asymptotiques sur $\mathrm{SP}_{3}$ et $\mathrm{SP}_{5}$ selon les deux scénarios indiqués ci- 
dessus ainsi que les gains génétiques induits sur la hauteur à cinq ans. Le gain génétique réalisable sur $\mathrm{HT}_{5}$ par sélection directe dans les mêmes conditions est également calculé. Outre les gains maximaux asymptotiques, calculés à partir des formules données dans ce paragraphe, sont calculés les gains génétiques correspondant à une sélection massale, une sélection sur test de descendance et une sélection combinée sur familles de pleins-frères. L'effectif retenu par famille de demi-frères ou de pleins-frères est de 50, ce qui correspond au minimum adopté jusqu'à présent dans les tests de descendances de pin maritime. Il est compatible avec des essais en serre ou des dispositifs spécialisés à l'extérieur.

On constate que le gain génétique réalisable sur $\mathrm{SP}_{3}$ dans le cas de la création d'une variété spécialisée va de $20 \%$ dans le cas de la sélection combinée à environ $50 \%$ pour la sélection sur test de descendance (valeur de $35 \%$ dans l'hypothèse moyenne). La perte génétique induite sur la hauteur à 5 ans par cette création variétale s'échelonne de $7 \%$ à $15 \%$. La valeur du gain génétique maximum réalisable sur $\mathrm{SP}_{3}$ par sélection sur test de descendance $(47,13 \%)$ est toutefois sensiblement inférieure au gain génétique asymptotique correspondant au même taux de sélection $(61,58 \%)$. En ce qui concerne la hauteur à 5 ans, la perte génétique causée par la sélection sur test de descendance $(14,67 \%)$ serait très inférieure à la perte que l'on aurait par contre-sélection directe sur ce caractère en test de descendance $(36,03 \%)$, résultat par ailleurs très proche de la valeur asymptotique $(39,14 \%)$. Compte tenu de la faible valeur prédictive de la hauteur à 5 ans vis-à-vis du volume adulte (Bergoin, 1993), une réduction de 20 à $50 \%$ de la valeur de $\mathrm{SP}_{3}$ reste compatible avec un gain génétique important sur le volume. Dans le cas du scénario d'une sélection faible dans la population d'amélioration pour la sensibilité à Melampsora en troisième année, les gains génétiques, si l'on prend en compte la possibilité d'une sélection massale, vont de 3 (sélection massale) à $15 \%$ (sélection combinée), selon la vraie valeur de l'héritabilité. Sur le plan pratique, seule la sélection combinée est réaliste, puisque la sélection pratiquée pourrait se faire sur les dispositifs ordinaires de gestion de la population d'amélioration, en profitant de l'opportunité d'attaques de rouille à un taux suffisant et en utilisant toute l'information disponible.

En ce qui concerne $\mathrm{SP}_{5}$, les résultats obtenus sont beaucoup plus favorables car ils montrent qu'il serait possible d'obtenir un gain génétique plus élevé pour la résistance à Melampsora sans incidence défavorable sur la hauteur juvénile. En éliminant la sélection massale, qui n'offre aucun intérêt, la stratégie de création d'une variété spécialisée permet alors d'obtenir, par sélection combinée ou sélection sur test de descendance, une résistance importante dans l'hypothèse minimale (gains de 53 ou $64 \%$ ), très forte dans l'hypothèse moyenne (gains de 75 ou $90 \%$ ) et quasi complète dans l' hypothèse maximale (gains de 94 ou $100 \%$ ). Le gain génétique corrélé sur la hauteur à 5 ans irait alors de 12 à $29 \%$, valeur proche de celle que l'on pourrait obtenir par sélection directe sur ce caractère, elle-même voisine de la valeur asymptotique. Pour la stratégie alternative (sélection dans la population d'amélioration), les gains génétiques sur $\mathrm{SP}_{5}$ iraient de 20 à $40 \%$ avec des gains corrélés sur $\mathrm{HT}_{5}$ de 4,5 à $11 \%$. Cette stratégie permettrait donc une réduction sensible de la sensibilité du pin maritime à la rouille avec, de surcroit, un effet bénéfique sur la vigueur juvénile.

On peut se demander dans quelle mesure les conclusions qui ont été formulées sur la base d'effectifs par famille de 50 individus devraient être révisées si l'on modifiait ces effectifs. La figure 1 donne, pour le caractère de sensibilité à Melampsora, l'évolution des coefficients de prédiction génétique généralisés pour des effectifs par famille de 25,50 et 100. Ces coefficients de prédiction génétique correspondent aux gains génétiques asymptotiques, à la sélection massale, à la sélection sur test de descendance et à la sélection combinée. L'héritabilité varie dans l'intervalle $[0,04 ; 0,42]$ qui correspond à l'ensemble des résultats des deux essais. Les gains génétiques relatifs sont évidemment proportionnels aux valeurs des $C P G$ qu'il suffit de multiplier par le coefficient de variation phénotypique, identique pour $\mathrm{SP}_{3}$ et $\mathrm{SP}_{5}$.

On constate que les gains génétiques prédits sont peu affectés par l'effectif par famille pour la sélection combinée, dans toute la plage de 


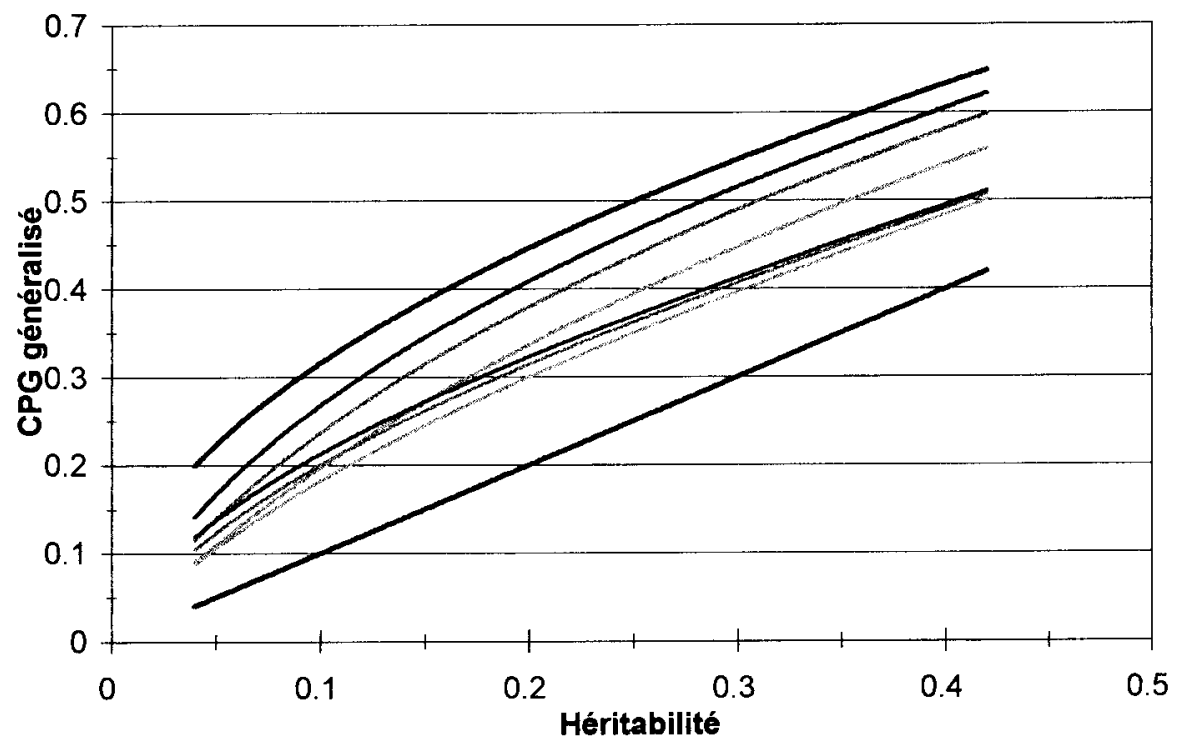

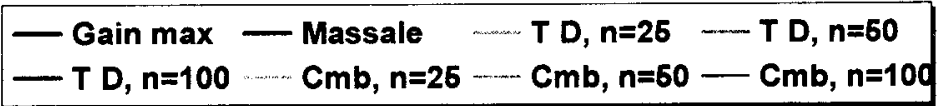

Fig 1. Évolution, en fonction de l'héritabilité de la sensibilité à Melampsora, des $C P G$ généralisés du caractère pour la sélection massale, la sélection sur test de descendance et la sélection combinée, avec des effectifs par famille de 25,50 et 100 individus. La courbe supérieure donne la valeur maximale du $C P G$; la courbe inférieure n'est autre que l'héritabilité du caractère (droite de pente 1). Les trois courbes immédiatement supérieures, pour $h^{2}>0,2$, correspondent à la sélection combinée $(\mathrm{Cmb})$. Les trois courbes suivantes concernent la sélection sur test de descendance (TD).

variation de l' héritabilité de la sensibilité. Lorsque l'héritabilité est supérieure à 0,4 , ils sont pratiquement identiques. Pour la sélection sur test de descendance, qui est toujours la plus efficace à effectif égal, l'incidence de l'effectif sur le gain génétique attendu est plus forte.

Toutefois, les deux courbes correspondant à $n=50$ et $n=100$ sont proches l'une de l'autre, alors que la courbe établie pour $n=25$ est plus nettement décalée vers le bas et se rapproche du faisceau des trois courbes de la sélection combinée.

Il apparaît donc que la sélection sur test de descendance, avec au moins 50 individus par famille, constituerait une méthode de sélection proche de l'optimum pour la création de variétés résistantes à Melampsora. La sélection com- binée, à un taux de l'ordre de $50 \%$ constituerait un outil plutôt orienté vers la sélection, à l'intérieur de la population d'amélioration, des parents à retenir pour passer à la génération suivante. Il est bien évident que l'on pourrait combiner ces deux méthodes à chaque cycle de sélection. La figure 2 schématise une possibilité d'intégration du critère de sensibilité à la rouille dans le programme d'amélioration du pin maritime des Landes. Elle tient compte de l'état d'avancement actuel de ce programme qui est arrivé au stade de la deuxième génération de la population d'amélioration mais où la génération $G_{2}$ ne représente qu'une partie seulement des descendants des arbres sélectionnables en $\mathrm{G}_{1}$. Dans ces conditions, le tri des individus les 


\section{Population d'amélioration}

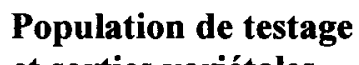

Génération et sorties variétales

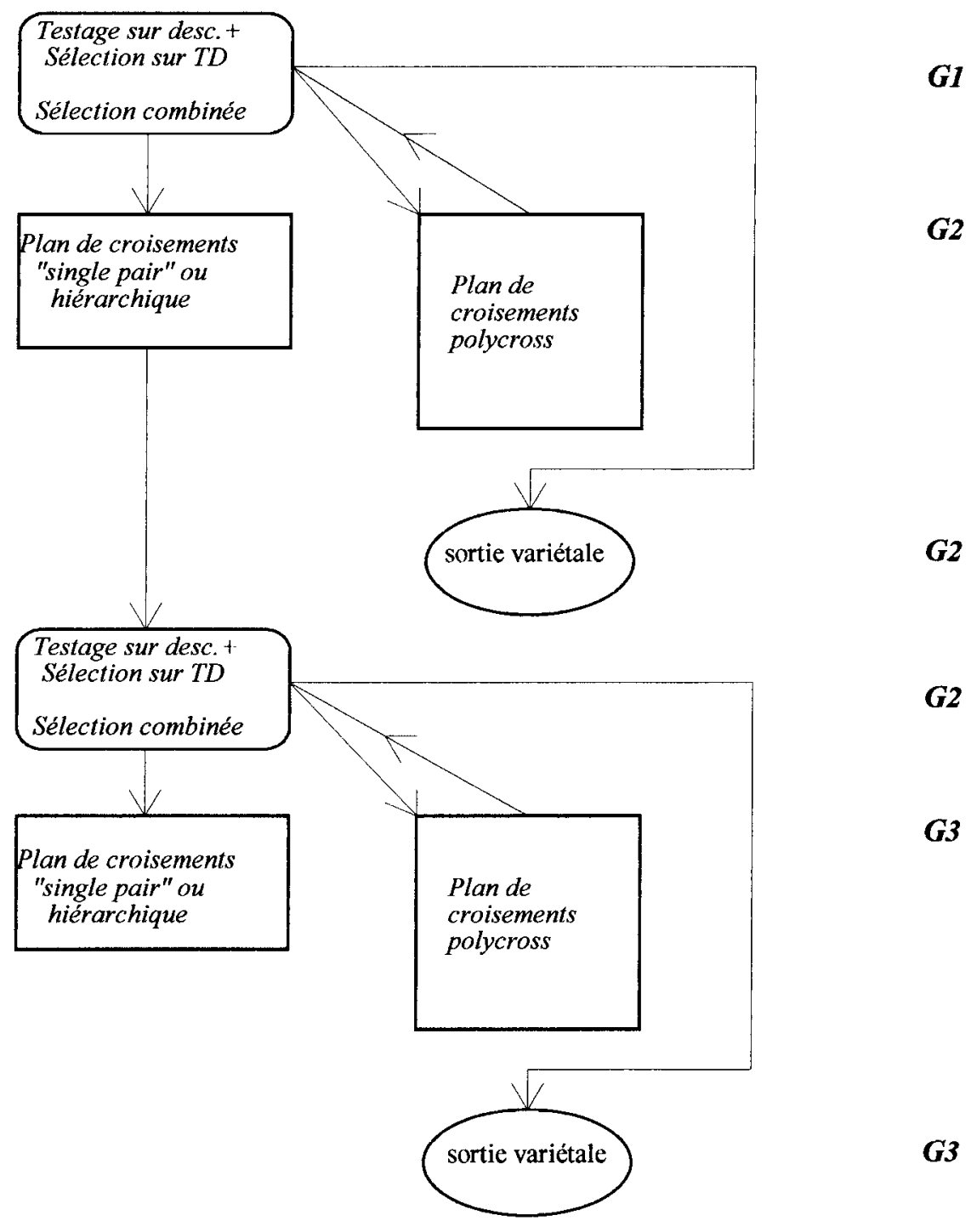

Fig 2. Modalité d'intégration du critère de sensibilité à Melampsora pinitorqua dans le programme d'amélioration du pin maritime des Landes. 
moins sensibles par sélection combinée serait réalisé, dans l'immédiat, en générations $G_{l}$ ou $\mathrm{G}_{2}$ et l'évaluation des performances des parents sur la base des observations réalisées sur les tests de descendances $\mathrm{G}_{2}$ ou $\mathrm{G}_{3}$.

\section{DISCUSSION}

Nous avons donc montré que le pourcentage de pousses présentant au moins un chancre est un caractère représentatif de la sensibilité globale à Melampsora d'un plant de pin maritime. Il intègre à la fois la probabilité d'attaque, I'intensité de l'attaque sur la flèche et la réaction de celle-ci. L'absence de corrélation entre réaction de la flèche à l'attaque et son diamètre peut sembler étonnante car on s'attendrait à ce que des pousses de fort diamètre réagissent mieux à un niveau donné d'attaque que des pousses plus grêles. En fait, le développement du mycélium est peut-être favorisé par une croissance en diamètre importante, ce qui conduirait à une compensation, les pousses les plus à même de réparer l'attaque étant aussi celles qui subissent l'agression la plus forte. L'absence de corrélation intraclasse significative de $\mathrm{DP}_{5}$ avec $\mathrm{SP}_{5}$ et $\mathrm{NCF}_{5}$ montre par ailleurs que l'explication ne réside pas dans une relation entre le nombre de basidiospores « interceptées » et la surface de la pousse, proportionnelle à son diamètre.

Sur le plan génétique, les résultats de cette étude montrent clairement que les paramètres exprimant la variabilité génétique de la sensibilité du pin maritime des Landes à Melampsora pinitorqua ne sont pas biaisés par l'existence d'effets maternels, du moins à partir de trois ans. Cette conclusion est très " robuste " car elle porte sur les descendants de 18 arbres non apparentés et sur deux essais installés et conduits indépendamment dans deux sites différents. Nous avons ensuite montré que le déterminisme génétique peut être considéré comme totalement additif. Ce résultat a des conséquences importantes sur la façon de gérer la population d'amélioration et d'envisager la création variétale. Un taux d'attaque trop faible pourrait être le facteur explicatif principal de l'absence de variabilité génétique significative pour la sensibilité à Melampsora à quatre ans. Les héritabilités des deux hauteurs à cinq ans sont très différentes. Celle du diallèle $6 \times 6$ correspond à la valeur habituelle que l'on a dans des tests de descendance de pin maritime de cet âge (Bergoin, 1993). En revanche, l'héritabilité dans le diallèle $12 \times 12$ est anormalement faible. Comme elle n'est pas associée à un coefficient de variation phénotypique plus élevé que dans le premier essai, il ne semble pas que cette faible valeur puisse être attribuée à une variabilité environnementale plus importante. On peut, en revanche, penser à des effets de concurrence très importants, dus, par exemple, à un désherbage trop tardif empêchant les différences entre génotypes de s'exprimer pleinement pour la croissance en hauteur. L'écart entre les deux héritabilités est donc dû essentiellement à une différence dans les variances d'AGC. Le tableau XI montre que c'est le même type d'explication qui vaut pour rendre compte de la différence d'héritabilité entre $\mathrm{SP}_{3}$ et $\mathrm{SP}_{5}$ : les valeurs de ces héritabilités sont à peu près dans le même rapport que les variances d'AGC. II semble donc que le degré de contrôle génétique de la sensibilité à la rouille du pin maritime des Landes augmente de la troisième à la cinquième saison de végétation, avec un changement de signe de la corrélation génétique avec la hauteur à cinq ans. Cette conclusion est renforcée par les résultats obtenus pour la sensibilité en quatrième année, $\mathrm{SP}_{4}$, qui présente une héritabilité et une corrélation génétique avec $\mathrm{HT}_{5}$ non significativement différentes de 0 . Le faible pourcentage de pousses attaquées en quatrième année $(11,37 \%)$, présenté dans le tableau III, peut être en partie responsable de la faible héritabilité de la sensibilité à cet âge. Comme hypothèse alternative pour expliquer l'évolution de l'héritabilité de la sensibilité et de sa corrélation génétique avec la hauteur à 5 ans, on pourrait envisager un effet physique de la croissance en hauteur sur la probabilité d'attaque des jeunes pousses. Ainsi, à partir d'un certain seuil, ces pousses se trouveraient au dessus du nuage de basidiospores ; elles ne pourraient plus alors être attaquées. L'âge agirait, dans ce cas, de façon indirecte sur la sensibilité à la rouille et non directement, en conditionnant l'évolution physiologique du plant et, notamment, la phénologie du début de croissance. Dans le ca- 
dre de cette deuxième hypothèse, les caractéristiques de la station et les façons culturales telles que l'époque de réalisation et la qualité des débroussaillages interviendraient de la même façon que l'âge sur le niveau d'héritabilité de la sensibilité et sa corrélation génétique avec la hauteur. Si cette hypothèse est vraie, on s'attendrait à une augmentation du coefficient de variation phénotypique de la sensibilité corrélative à un accroissement de la hauteur moyenne des plants au moment de l'attaque. De fait, le coefficient de variation phénotypique de $\mathrm{SP}_{5}$ $(0,90)$ est légèrement supérieur à celui de $\mathrm{SP}_{3} 0$, 83). Nos résultats ne sont toutefois pas suffisants pour permettre de choisir entre ces deux explications.

Une influence directe de l'âge sur la corrélation génétique entre hauteur et sensibilité à $M e$ lampsora serait en faveur d'une notation du degré d'attaque, dans les plantations comparatives, en quatrième ou en cinquième saison de végétation de préférence à la seconde ou à la troisième. En revanche, il est évident que des tests en serre ou en chambre climatique, permettant un contrôle des stades phénologiques, devraient être réalisés à un ou deux ans. Les résultats de Martinsson sur pin sylvestre ( 1980 et 1987) vont tout à fait dans ce sens puisqu'ils mettent en évidence une bonne corrélation des estimations de sensibilité à Melampsora à différents stades de développement, de 2 mois jusqu'à 7 ans. Il ressort par ailleurs clairement de l'évaluation des gains génétiques possibles que le critère de sensibilité à Melampsora serait à prendre en compte au niveau de la gestion de la population d'amélioration, sans qu'il soit besoin d'installer des essais spécialisés, et en profitant simplement d'attaques spontanées afin de sélectionner avec une intensité de sélection modérée, les plants les moins atteints. Le gain génétique relatif minimal à cinq ans en sélection combinée $(20 \%)$ serait suffisant pour justifier des campagnes de notations peu coûteuses par rapport aux autres dépenses engagées pour le programme d'amélioration. Il serait intéressant d'évaluer la relation entre degré d'attaque à trois ans ou à cinq ans et déformation de la bille de pied qui constitue la majeure partie de la valeur de l'arbre lors de la dernière éclaircie et de la coupe rase. Cela permettrait de mieux évaluer l'impact économique d'un gain génétique sur la résistance à la rouille. Nos résultats montrent par ailleurs que l'on peut espérer créeer des variétés très résistantes à la rouille. Le coût des investissements à engager pour obtenir ce résultat est évidemment non négligeable. Il serait important si l'on réalisait à grande échelle des essais spécialisés avec inoculation artificielle. Il serait beaucoup plus modique si l'on intégrait simplement le critère de sensibilité dans le suivi des tests de descendances polycross qui constituent actuellement la population de testage dans le programme d'amélioration du pin maritime en Aquitaine. L'essentiel du coût de création des variétés résistantes serait alors induit par la phase de multiplication en masse des génotypes sélectionnés : croisements contrôlés s'il s'agit de « variétés bulk o obtenues par interpollinisation de géniteurs sélectionnés ou de l'installation d'un verger à graines d'équivalents-clones spécialisé. L'absence, de variance d'effets maternels ou d'aptitude spécifique à la combinaison rend aisée l'acquisition de nouveaux résultats permettant d'affiner l'estimation de I'héritabilité de la sensibilité à Melampsora et de son coefficient de prédiction génétique avec la hauteur juvénile. En effet, ces deux paramètres sont essentiels pour évaluer l'impact économique de l'intégration de la sélection pour la résistance à la rouille dans le programme d'amélioration du pin maritime. Compte tenu du mode d'hérédité totalement additif, toutes les observations réalisées sur de jeunes tests de descendances, y compris des tests de demi-frères, fourniront une information utile. L'ensemble de ces informations, avec l'estimation de l'impact économique des dégâts, permettrait d'évaluer les moyens qu'il serait raisonnable de consacrer à une sélection du pin maritime des Landes pour une résistance plus ou moins grande à Melampsora pinitorqua.

\section{CONCLUSION}

L'analyse génétique approfondie de la variabilité de la sensibilité à la rouille courbeuse du pin maritime d'origine landaise fait donc apparaître que ce critère mériterait d'être inclus dans le programme d'amélioration de l'espèce. Le 
mode d'hérédité, complètement additif, et l'héritabilité relativement faible du caractère traduisant la sensibilité devraient conduire à privilégier la sélection sur test de descendance pour obtenir les gains génétiques les plus élevés au niveau de la création variétale. La sélection pour la résistance à Melampsora pinitorqua pourrait également être mise en œuvre au niveau de la gestion de la population d'amélioration, avec une intensité de sélection beaucoup plus faible. Le crible des géniteurs utilisés pour les sorties variétales serait alors suivi de la production, par croisements contrôlés, de « variétés bulk », directement utilisables pour les reboisements par plantation, ou pour la production de matériel de base pour des vergers d'équivalents-clones (Baradat, 1987). Dans ce deuxième cas, les variétés résistantes pourraient être utilisées, avec un délai plus long, dans les reboisements par semis direct. On peut donc envisager d'intégrer à chaque cycle de sélection le critère de sensibilité à Melampsora, à quatre ou cinq ans, avant de prendre en compte les caractères habituels de croissance, de forme et de résistance aux insectes, qui peuvent raisonnablement faire l'objet d'une sélection à dix ans. Si l'on envisage d'investir beaucoup pour obtenir des variétés résistantes, le testage des parents sur descendance pourrait prendre la forme soit d'essais en serre, soit d'essais spécialisés à l'extérieur. On prévoirait, dans un cas comme dans l'autre, une inoculation artificielle sur un ou deux ans afin d'obtenir une infestation des jeunes plants aussi uniforme que possible. Cette procédure, qui ressemblerait à celle suivie dans le programme coopératif des pins du sud-est des États-Unis, Pinus taeada et Pinus elliottii(Anderson et Powers, 1985 ; Powers et Kraus, 1983 ; de Souza et al, 1992), n'allongerait pas la durée d'un cycle de sélection. En effet, elle s'opérerait en parallèle avec la mise en place et le suivi des essais permettant une sélection sur les autres caractères pris en compte dans le programme d' amélioration du pin maritime.

\section{RÉFÉRENCES}

Anderson RL, Powers HR Jr (1985) The resistance screening center: screening for disease as a service for tree improvement programs. Proceedings of
Rusts of Hard Pines IUFRO working party Conference (J Barrows-Broaddes, R Powers, eds), University of Georgia, Athens, GA, États-Unis, 59-63

Baradat $\mathrm{P}$ (1976) Use of juvenile-mature relationships in individual selection including information from relatives. Proceeding of IUFRO Meeting on advanced generation breeding, Bordeaux, 12 I-138

Baradat P (1987) Méthode d'évaluation de la consanguinité chez les plants issus de vergers à graines de semis de première génération. II. Les vergers d'équivalents-clones. Silvae Genetica 36, 134-144

Baradat $P$ (1989) Amélioration génétique des arbres forestiers, éléments méthodologiques. Laboratoire d'amélioration des arbres forestiers, Inra, Bordeaux, $204 \mathrm{p}$

Baradat $\mathrm{P}$, Labbé $\mathrm{T}$ (1995) Opep. Un logiciel intégré pour l'amélioration des plantes pérennes. Traitement statistique des essais de sélection. Éd Cirad, Montpellier, 303-330

Baradat P, Labbé T, Bouvet JM (1995a) Conception d'index pour la sélection réciproque récurrente. Aspects génétiques, statistiques et informatiques. Traitement statistique des essais de sélection. Séminaire biométrie et génétique. Éd Cirad, Montpellier, 101-150

Baradat P, Maillart M, Marpeau A, Slak MF, Yani A, Pastuszka P (1995b) Utility of terpenes to assess population structure and mating patterns in coni. fers. Population genetics and genefic conservation of forest trees (P Baradat, WT Adams, G Müller-Starck, eds), SPB Academic Publishing, Amsterdam, Pays-Bas, 5- 27

Baradat P, Marpeau-Bezard A (1988) Le pin maritime. Biologie et génétique des terpènes pour la connaissance et l'amélioration de l'espèce. Thèse doctorat d'État, univ Bordeaux-I, $551 \mathrm{p}$

Baradat P, Pastuszka P (1992) Le pin maritime. «Amélioration des espèces végétales cultivées», Éditions Inra, Paris, 695-709

Becker WB (1984) Manual of Quantitative Genetics. Academic Enterprises, Pullman, États-Unis, 188 p

Bedard PR, Hsu CS, Spangelo LPS, Rousselle G (1971) Genetic, phenotypic, and environmental correlations among 28 fruit and plant characters in the cultivated strawberry. Can J Genet Cytol 13, 470-479

Bergoin A (1993) Efficacité de la sélection réalisée en forêt et en test de descendance chez le pin maritime. Prédiction du gain génétique sur le volume à partir des composantes de la hauteur. DEA, université de Nancy-I, $36 \mathrm{p}$

Cockerham CC (1963) Estimation of Genetic Variances. Stat Genetics and Plant Breeding, NAS-NRC 982, 53-94 
Desprez ML (1980) Melampsora pinitorqua. II. Partie expérimentale. Mémoire de DEA, Ina-PG, 14 p

Desprez-Loustau ML, Baradat P (1991) Variabilité interraciale de la sensibilité à la rouille courbeuse chez le pin maritime. Ann Sci For 48, 497-511

Desprez-Loustau ML, Dupuis F (1994) Variation in the phenology of shoot elongation between geographic provenances of maritime pine - Implication for the synchrony with the phenology of the twisting fungus. Ann $S_{c i}$ For 51, 553-568

Falconer DS (1981) Introduction to Quantitative Genetics, $2^{e}$ edition. Longman, New York, NY, ÉtatsUnis, $340 \mathrm{p}$

Gallais A (1973) Sélection pour plusieurs caractères. Synthèse critique et généralisation. Ann Amélior Plantes 23, 183-208

Gallais A (1990) Théorie de la sélection en amélioration des plantes. Masson, Paris, $588 \mathrm{p}$

Garretsen F, Keuls M (1977) A general method for the analysis of genetic variation in complete and incomplete dialleles and North Carolina 2 designs. Part 1. Procedures and general formulas for the random model. Euphytica 26, 537-551

Griffing B. (1966) Concept of general and specific combining ability in relation to diallele crossing systems. Aust Journ Biol Sci 9, 463-493

Illy G (1966) Recherches sur l'amélioration génétique du pin maritime. Ann Sci For 23, 757-948

Janssens MJJ (1979) Co-heritability: its relation to correlated response, linkage and peiotropy in case of polygenic inheritance. Euphytica 28, 601-608

Keuls M, Garretsen F (1978) A general method for the analysis of genetic variation in complete and incomplete dialleles and North Carolina 2 designs. Part II. Procedures and general formulas for the fixed model. Euphytica 27, 49-68

Klingström A (1969) Melampsora pinitorqua (Braun) Rostr on progenies of Pinus silvestris $\mathrm{L}$ and in relation to growth regulating substances. Studia Forestalia Suecica $69,1.75$

Lebart L, Morineau A, Fénelon JP (1979) Traitement des données statistiques. Dunod, Paris, $510 \mathrm{p}$
Maillart M (1993) Étude de la structuration génétique et du régime de reproduction d'un peuplement de pin maritime issu de régénération naturelle. DEA, université de Nancy-I, $32 \mathrm{p}$

Martinsson $\mathrm{O}$ (1975) Test of resistance against parasitic fungi on forest trees. Sveriges Skogsvardsf. Tidster 73, 31-46

Martinsson O. (1980) Testing scots pine for resistance to pine twist rust. Folia Forestalia Suecica 422, 25-31

Martinsson O (1987) Scots pine resistance to pine twist rust. Conformity between the resistance found in an artificial environment and field trials. Silvae Genetica 35, 15-21

Mode CG, Robinson HF (1959) Pleiotropism and the genetic variance and covariance. Biometrics 15 , 518-537

Nei M (1960) Studies on the application of biometrical genetics to plant breeding. Memoirs of the College of Agriculture, Kyoto University ( $\left.\mathrm{n}^{\circ} 32\right), 100 \mathrm{p}$

Powers HR Jr, Kraus JF (1983) Developing fusiform rust-resistant loblolly and slash pines. Plant Dis 67, 187-189

Rao TS, Goud JV (1971) Recent trends in Rice breeding in Myosore State. IV Correlations, coheritability and selection indices for yield and yield coponents in three environments. Z Pflanzenzüchtg $65,125-128$

Saporta G (1990) Probabilité, analyse des données et statistiques. Technip, Paris, $493 \mathrm{p}$

Searle SR (1971) Linear models. John Wiley, New York, $532 \mathrm{p}$

Snedecor GW, Cochran WG (1967) Statistical Methods (6th edition). The Iowa State Univ, Ames, IA, États-Unis, 593 p

de Soura SN, Hodge GR, White TL (1992) Indirect prediction of breeding values for fusiform rust resistance of slash pine parents using greenhouse tests. For $S_{c i}$ 38, 45-60

Weissenberg K (1978) Variation in host resistance and pathogen agressivity in the Pinus silvestris-Melampsora pinitorqua system. 3rd Int Congr of Plant Pathol, Munich, 16•23 août 1978, 293 p 


\section{ANNEXE \\ Calcul des composantes de la variance ou de la covariance en modèle factoriel sans utilisation du niveau individuel.}

Les analyses de variance en classification croisée et en modèle mixte (effet bloc fixé et effet famille aléatoire) ont été réalisées selon le modèle « Henderson III » (Searle, 1971), avec une variante proposée par la librairie Opep (Baradat, 1989 ; Baradat et Labbé, 1995) permettant d'estimer les composantes individuelles de la variance ou de la covariance, en l'absence d'interaction, sans utiliser les sommes de carrés ou de coproduits intracellule. Cette modification est bien adaptée au traitement de variables discrètes, par exemple de type 0 -1. Le principe en est le suivant pour un modèle mixte en classification croisée, $y_{i j k}=\mu+a_{i}+\beta_{j}+(a \beta)_{i j}+e_{i j k}$ où $a_{i}$ est un effet aléatoire et $\beta_{j}$ un effet fixé. En présence d'interaction, l'estimation des trois variances des variables aléaloires, $\sigma_{a,}^{2}, \sigma^{2}{ }_{(\alpha \beta)}$ et $\sigma_{e}^{2}$, est réalisée par résolution du système de trois équations donné ci-dessous :

$$
\begin{gathered}
\hat{\sigma}_{e}^{2}=\left(T_{o}-T_{A B}\right) /(N \cdot C) \\
\left.\hat{\sigma}_{(a \beta)}^{2}=\left[T_{A B}-R_{(} \mu, \alpha, \beta\right)-(C-A-B+1) \hat{\sigma}_{e}^{2}\right] / k_{1} \\
\hat{\sigma}_{a}^{2}=\left[T_{A B}-T_{B}-(C-B) \hat{\sigma}_{e}^{2}\right] / k_{2}-\hat{\sigma}_{(a \beta)}^{2}
\end{gathered}
$$

où $T_{0}$ est la somme des carrés totale,

$$
\sum_{i=1}^{A} \sum_{j=1}^{B} \sum_{k=1}^{n_{i j}} y_{i j k}^{2} \text {; }
$$

$T_{A B}$ est la somme des carrés entre les $C$ cellules :

$$
\sum_{i=1}^{A} \sum_{j=1}^{B} y_{i j .}^{2} / n_{i j}
$$

$N$ est le nombre total d'individus ; $A$ est le nombre de modalités du facteur $a$;

$$
T_{B}=\sum_{j=1}^{B} y_{i j}^{2} / n_{j} ;
$$

est la somme des carrés entre les $B$ modalités du facteur $\beta ; R(\mu, \alpha, \beta)$ est la somme des carrés expliquée par la moyenne générale, $\mu$, et les effets principaux des deux facteurs, le facteur aléatoire, $a$, étant considéré provisoirement comme fixé. On a :

$$
\begin{gathered}
R(\mu, \alpha, \beta)=T_{A}-t_{B} \\
\text { où } t_{B}=[r]^{\prime}[C]^{\cdot 1}[r]
\end{gathered}
$$

Les éléments du vecteur colonne $[r]$ de dimen$\operatorname{sion}(B \cdot 1,1)$ sont :

$$
r_{i}=y_{i j}-\sum_{i=1}^{\lambda} n i j \bar{y}_{i .}
$$

Les éléments de la matrice $[C]$, carrée et de dimensions $(B \bullet 1, B \bullet 1)$ sont :

$$
\begin{gathered}
c_{i j}=n_{i}-\sum_{i=1}^{A} n_{i j}^{2} / n_{i .} \text { (éléments diagonaux) } \\
c_{j j^{\prime}}=-\sum_{i=1}^{A} n_{i j} n^{\prime}{ }_{i j} / n_{i .} \text { (éléments non diagonaux) } \\
k_{1}=N-K \text { avec } K=\sum_{i=1}^{A} \lambda_{i}+\operatorname{tr}\left([C]^{-1} \sum_{i=1}^{A} \Phi_{i}\right)
\end{gathered}
$$
où :

$$
\lambda_{i}=\sum_{i=1}^{B} n_{i j}^{2} / n_{i}
$$

et :

$$
\begin{gathered}
\Phi_{i, j j}=\left(n_{i j}^{2} / n_{i}\right)\left(\lambda_{i}+n_{i}-2 n_{i j}\right) \\
\Phi_{i, j j}=\left(n_{i j} n_{i j} / n_{i}\right)\left(\lambda_{i}-n_{i j}-n_{i j}\right) \\
k_{2}=N-\sum_{j=1}^{A}\left(\sum_{i=1}^{A} n_{i j}^{2}\right) / n_{i j}
\end{gathered}
$$

Si $\sigma_{(2, \beta)}^{2}=0$, le système à résoudre se réduit à :

$$
\begin{gathered}
\hat{\sigma}_{a}^{2}=\left[T_{A B}-R(\mu, \alpha, \beta)\right] / k_{1} \\
\hat{\sigma}_{a=}^{2}=\left[T_{A B}-T_{B}-(C-B) \hat{\sigma}_{e}^{2}\right] / k_{2}
\end{gathered}
$$

Et l'on évite ainsi d'utiliser la somme de carrés intracellule qui est la seule à prendre en compte les effets individuels, à distribution discontinue. Le même principe d'estimation vaut pour les covariances en remplaçant les sommes de carrés par les sommes de coproduits correspondantes et en modifiant le calcul de $t_{B}$ où les vecteurs $[r]$ et $[r]$ ' doivent concerner chacun l'un des deux caractères $l$ et $l$ ' dont on veut calculer la covariance. Cette méthode est plus précise qu'une analyse de variance sur moyennes de cellules puisqu'elle tient compte des différences d'effectifs et utilise ainsi toute l'information disponible. 